AEI-2010-140

\title{
ASYMPTOTIC SYMMETRIES OF THREE-DIMENSIONAL GRAVITY COUPLED TO HIGHER-SPIN FIELDS
}

\author{
A. Campoleoni, S. Fredenhagen, S. Pfenninger and S. Theisen \\ Max-Planck-Institut für Gravitationsphysik \\ Albert-Einstein-Institut \\ Am Mühlenberg 1 \\ 14476 Golm, GERMANY \\ andrea.campoleoni@aei.mpg.de,stefan.fredenhagen@aei.mpg.de, \\ stefan.pfenninger@aei.mpg.de, stefan.theisen@aei.mpg.de
}

\begin{abstract}
We discuss the emergence of $\mathcal{W}$-algebras as asymptotic symmetries of higher-spin gauge theories coupled to three-dimensional Einstein gravity with a negative cosmological constant. We focus on models involving a finite number of bosonic higher-spin fields, and especially on the example provided by the coupling of a spin-3 field to gravity. It is described by a $S L(3) \times S L(3)$ Chern-Simons theory and its asymptotic symmetry algebra is given by two copies of the classical $\mathcal{W}_{3}$-algebra with central charge the one computed by Brown and Henneaux in pure gravity with negative cosmological constant.
\end{abstract}




\section{Contents}

1 Introduction $\quad 2$

2 Coupling to gravity and the Chern-Simons action 5

2.1 Free theory . . . . . . . . . . . . . . . . . 5

2.2 Chern-Simons formulation . . . . . . . . . . . . . . . 8

2.3 The spin-3 example . . . . . . . . . . . . . . . . . . 13

3 Review of Chern-Simons theory with boundary $\quad 16$

4 Asymptotic symmetries $\quad 20$

4.1 Boundary conditions . . . . . . . . . . . . . . . . . 20

4.2 Asymptotic symmetry algebra . . . . . . . . . . . . . . . . 23

4.3 Fall-off conditions for the metric-like fields . . . . . . . . . . . . . . . 27

5 Comments on higher rank groups $\quad 30$

6 Conclusions $\quad 32$

$\begin{array}{ll}\text { A Conventions } & 33\end{array}$

B Useful formulae $\quad 35$

\section{Introduction}

The covariant description of the free propagation of massless higher-spin particles in a four-dimensional flat background was obtained long ago by Fronsdal [1] in terms of gauge theories. However, it was soon realised that the coupling to gravity of the free actions displays various pathologies. ${ }^{1}$ For instance, Aragone and Deser [6] showed the inconsistency of the minimal coupling of higher-spin gauge fields to gravity. At its heart the obstruction rests on the impossibility to preserve the invariance of the free action under higher-spin gauge transformations at the interacting level. In fact, the gauge variation of the minimally coupled higher-spin actions is proportional to the full Riemann tensor. As such, it cannot be cancelled by any variation of the Einstein-Hilbert action. The highest-

\footnotetext{
${ }^{1}$ For a review of the old no-go arguments and of the more recent results on how higher-spin interactions in $D \geq 4$ can nevertheless be constructed, we refer the reader to [2]. Other reviews on various aspects of higher-spin gauge theories can be found in $[3,4,5]$.
} 
spin which is allowed is $3 / 2$ which leads to supergravity. There the gauge variation of the Rarita-Schwinger action is proportional to the Ricci tensor rather than to the Riemann tensor, and this is one of the crucial conditions allowing supersymmetry.

In three space-time dimensions the situation is very different: on the one hand, Fronsdal's gauge fields with "spin" $s>1$ do not propagate any local degree of freedom. ${ }^{2}$ On the other hand, in $D=3$ the Weyl tensor vanishes for any gravitational background and this suggests a possible way to avoid the no-go results for minimal coupling. This expectation was indeed confirmed by Aragone and Deser in [7]. However, this is not the unique example of consistent interactions for higher-spin gauge fields: a long-term effort by Vasiliev provided an interacting theory for an infinite tower of massless higher-spin fields in constant curvature backgrounds of any dimension [8]. On the other hand, the three-dimensional peculiarities offer interesting toy models without many of the technical complications that emerge when dealing with higher-spin fields in $D>3$. For instance, in [7] it was realised that in three dimensions there is no need to consider an infinite number of higher-spin fields in order to obtain consistent interactions.

For a long time this result was part of a collection of three-dimensional curiosities, like the by now well-known result of Brown and Henneaux [9] on the asymptotic symmetries of three-dimensional pure gravity with a negative cosmological constant. These authors first proved that - when considering asymptotically Anti-de Sitter spaces - the group of asymptotic symmetries is the conformal group in two dimensions. They also showed that its canonical realisation in terms of Dirac brackets of global charges possesses a central extension. While the emergence of the conformal group in two dimensions can also be inferred from the structure of the AdS conformal boundary, the latter observation was rather unexpected. Later on the central charge identified by Brown and Henneaux was shown to play a crucial role in possible microscopic interpretations of the entropy of the BTZ black hole [10, 11]. More generally, in modern terms the Brown-Henneaux results should be considered as precursors of the $A d S_{3} / C F T_{2}$ correspondence.

In the present paper we extend the considerations of Brown and Henneaux from the pure spin-2 case to the more general setup were also fields with spin $s>2$ are present. We then show how the coupling of these higher-spin fields to three-dimensional gravity allows for an enhancement of the boundary conformal symmetry. In fact, in presence of a negative cosmological constant the coupled system displays in general an extended conformal symmetry acting on the space of asymptotically AdS solutions of the field equations. In three space-time dimensions Einstein gravity can thus be considered as the simplest example of a wide class of higher-spin gauge theories whose dynamics is described by a conformal field theory on the boundary. Even if we shall not deal with the details of the boundary theory, the results we are going to present set the stage for possible higher-spin extensions of the standard $A d S_{3} / C F T_{2}$ correspondence.

\footnotetext{
${ }^{2}$ The notion of spin we are referring to is not related to the labelling of the representations of the little group, which becomes trivial for massless particles in $D=3$. It is simply associated to the transformation properties under Lorentz transformation of the fields we are going to consider.
} 
We analyse the structure of asymptotic symmetries mainly by focussing on the coupling of a spin-3 field to gravity with a negative cosmological constant. In this example the asymptotic symmetries are given by two copies of the classical Zamolodchikov $\mathcal{W}_{3}$-algebra with a central charge

$$
c=\frac{3 l}{2 G}
$$

coinciding with the Brown-Henneaux one [9]. We also comment on the relation between more general $\mathcal{W}$-algebras (see [12] for a review on $\mathcal{W}$-algebras) and the asymptotic symmetries of higher-spin gauge theories with richer spectra. Our discussion rests on another important observation on the three-dimensional world: the option to describe interactions of fields with spin $s>1$ by means of a Chern-Simons (CS) action. This was realised in $[13,14]$ for (super)gravity theories. In [15] Blencowe then proposed a Chern-Simons action which describes an infinite tower of interacting higher-spin fields. Blencowe's theory was then shown to belong to a one-parameter family of "topological" higher-spin interacting theories with unbounded spectra [16]. This rich set of gauge theories was further analysed by Vasiliev and collaborators (see [17, 18] and references therein), who also discussed their coupling to matter. On the other hand, as we already pointed out, in three space-time dimensions there is no need to consider an infinite tower of higher-spin fields to consistently switch on interactions. The possibility of describing them via a CS action is not a peculiarity of Blencowe's theory or its generalisations. It also applies to gauge theories with a finite number of higher-spin fields, and in particular to our spin-3 example.

In Section 2 we show how to cast generic higher-spin gauge theories in a Chern-Simons form. This requires a reformulation of the higher-spin dynamics along the lines of the frame formalism of gravity and the identification of a suitable gauge algebra. We discuss this last point in detail in the spin-3 case, for which we single out the $S L(3) \times S L(3)$ gauge group. We also show how this example fits in the class of $S L(n) \times S L(n)$ CS theories, that describes interactions between a group of fields where each integer spin between 2 and $n$ appears once. This formalism enables us to discuss asymptotic symmetries as global symmetries of a CS theory subject to proper boundary conditions. For this reason in Section 3 we review some general results on CS theories on manifolds with boundaries. They provide the basis of Section 4, where we resume the spin-3 example. We identify the precise set of boundary conditions which characterise the asymptotically Anti-de Sitter solutions of the field equations. We then derive the asymptotic symmetries which they imply and obtain a centrally extended classical $\mathcal{W}_{3} \otimes \mathcal{W}_{3}$ algebra. ${ }^{3}$ At the end of Section 4 we return to the metric-like formulation. First we fix the relation between metric-like fields and their frame-like counterparts at the non-linear level. Then we use this result to translate the boundary conditions for the CS theory in terms of fall-off conditions for the metric-like fields, thus enabling a more direct comparison with the standard BrownHenneaux results. Finally, in Section 5 we comment on the case of a general gauge group by comparing our boundary conditions with those implementing the Hamiltonian reduction of Wess-Zumino-Witten (WZW) models to Toda theories [21]. In particular,

\footnotetext{
${ }^{3}$ See $[19,20]$ for other connections between CS theories and $\mathcal{W}$-algebras.
} 
we point out the universal character of the Brown-Henneaux central charge (1.1), that emerges in a wide class of higher-spin gauge theories including the $S L(n) \times S L(n)$ example. Section 6 closes this paper with a brief summary of our results. In two appendices we specify our conventions and collect some useful formulae.

We have been informed by Marc Henneaux that he and Soo-Jong Rey have also studied the issue of asymptotic symmetry algebras of higher-spin theories in three dimensions [22].

\section{Coupling to gravity and the Chern-Simons action}

In this section we first recall some standard facts on the free theory for higher-spin gauge fields in $D \geq 4$. Recognising that the structure of the field equations does not depend on $D$, we then clarify our notion of higher-spin fields in $D=3$. Afterwards, starting from the frame-like description of the free theory, we show how in $D=3$ higher-spin gauge fields can be coupled to gravity via a Chern-Simons action. We then provide a class of examples by focussing on the coupling of a tower of fields of increasing spin $2,3, \ldots, n$, that is described by a $S L(n) \times S L(n)$ CS theory. We close this section describing in detail the simplest model of this class, that features the coupling of a spin-3 gauge field to gravity with a negative cosmological constant.

\section{$2.1 \quad$ Free theory}

In a Minkowski background of arbitrary dimension $D \geq 4$ the free propagation of a bosonic massless spin- $s$ particle can be described via a fully symmetric rank- $s$ tensor $\varphi_{\mu_{1} \ldots \mu_{s}}$ satisfying the second-order field equation [1]

$$
\mathcal{F}_{\mu_{1} \ldots \mu_{s}} \equiv \square \varphi_{\mu_{1} \ldots \mu_{s}}-\partial_{\left(\mu_{1} \mid\right.} \partial^{\lambda} \varphi_{\left.\mid \mu_{2} \ldots \mu_{s}\right) \lambda}+\partial_{\left(\mu_{1}\right.} \partial_{\mu_{2}} \varphi_{\left.\mu_{3} \ldots \mu_{s}\right) \lambda}{ }^{\lambda}=0,
$$

where here and in the following a pair of parentheses denotes a complete symmetrisation of the indices it encloses, with the minimum possible number of terms and without any normalisation factor. Eq. (2.1) is left invariant by the gauge transformation

$$
\delta \varphi_{\mu_{1} \ldots \mu_{s}}=\partial_{\left(\mu_{1}\right.} \xi_{\left.\mu_{2} \ldots \mu_{s}\right)}
$$

with a traceless gauge parameter:

$$
\xi_{\mu_{1} \ldots \mu_{s-3} \lambda}{ }^{\lambda}=0
$$

Notice that in the spin-2 case $\mathcal{F}_{\mu \nu}$ is the linearised Ricci tensor, and the transformation (2.2) is a linearised diffeomorphism. Imposing the double-trace constraint

$$
\varphi_{\mu_{1} \ldots \mu_{s-4} \lambda \rho^{\lambda \rho}}=0
$$


one can build a second-order Lagrangian that, up to total derivatives, is invariant under the constrained gauge transformations (2.2). The resulting action, identified by Fronsdal $[1]$, is

$$
S=\frac{1}{2} \int d^{D} x \varphi^{\mu_{1} \ldots \mu_{s}}\left(\mathcal{F}_{\mu_{1} \ldots \mu_{s}}-\frac{1}{2} \eta_{\left(\mu_{1} \mu_{2}\right.} \mathcal{F}_{\left.\mu_{3} \ldots \mu_{s}\right) \lambda}{ }^{\lambda}\right),
$$

and leads to field equations that are equivalent to (2.1). Gauge invariance fixes eq. (2.5) up to a normalisation factor [23]. For more details see the reviews collected in [4].

In an AdS background one can look for field equations which are invariant under the gauge transformations

$$
\delta \varphi_{\mu_{1} \ldots \mu_{s}}=\nabla_{\left(\mu_{1}\right.} \xi_{\left.\mu_{2} \ldots \mu_{s}\right)}
$$

where $\nabla_{\mu}$ is the AdS covariant derivative. They describe the propagation of the same number of degrees of freedom as a massless spin-s field in flat space [24]. However, covariant derivatives no longer commute: denoting by $l$ the AdS radius and by $g_{\mu \nu}$ the AdS metric one obtains

$$
\left[\nabla_{\mu}, \nabla_{\nu}\right] V_{\rho}=\frac{1}{l^{2}}\left(g_{\nu \rho} V_{\mu}-g_{\mu \rho} V_{\nu}\right)
$$

Therefore, one has to add extra terms to the field equations in order to keep the gauge invariance. The result is

$$
\mathcal{F}_{\mu_{1} \ldots \mu_{s}}-\frac{1}{l^{2}}\left\{\left[s^{2}+(D-6) s-2(D-3)\right] \varphi_{\mu_{1} \ldots \mu_{s}}+2 g_{\left(\mu_{1} \mu_{2}\right.} \varphi_{\left.\mu_{3} \ldots \mu_{s}\right) \lambda^{\lambda}}\right\}=0
$$

Here $\mathcal{F}$ denotes the combination entering eq. (2.1), with the substitution $\partial_{\mu} \rightarrow \nabla_{\mu}[24]$. Eq. (2.8) is gauge invariant only if the gauge parameter satisfies eq. (2.3). In order to relate these equations to an action principle it is convenient to introduce the deformed Fronsdal operator $\widehat{\mathcal{F}}$, defined by the left-hand side of eq. (2.8). In fact, imposing the double-trace constraint (2.4), field equations equivalent to (2.8) follow from the action

$$
S=\frac{1}{2} \int d^{D} x \sqrt{-g} \varphi^{\mu_{1} \ldots \mu_{s}}\left(\widehat{\mathcal{F}}_{\mu_{1} \ldots \mu_{s}}-\frac{1}{2} g_{\left(\mu_{1} \mu_{2}\right.} \widehat{\mathcal{F}}_{\left.\mu_{3} \ldots \mu_{s}\right) \lambda}{ }^{\lambda}\right)
$$

Even in this case, its structure is fixed by the request of gauge invariance (up to a conventional ordering choice for the covariant derivatives) [24].

In $D=3$ the little group of massless particles is the direct product of the multiplicative group $\{1,-1\}$ with $\mathbb{R}[25]$. As a result, excluding representations with continuous spin, one is left only with the two inequivalent representations of $\{1,-1\}$. The usual notion of spin in $D=3$ thus just reduces to a distinction between bosons and fermions [25]. Nevertheless, one can still consider the field equations (2.1) or (2.8) for tensors of arbitrary rank. They force on-shell the propagation of a number of local degrees of freedom equal to the number of components of a traceless tensor of the same rank in $D-2$ dimensions [4]. Therefore, in $D=3$ they do not lead to the propagation of any local degree of freedom if the rank of the tensor is greater than one. However, even if the bulk dynamics is trivial, in presence of a cosmological constant fields with different rank lead to different boundary 
dynamics. This distinction motivates to denote as spin the rank $s$ of the field $\varphi_{\mu_{1} \ldots \mu_{s}}$. An higher-spin gauge field in $D=3$ is thus a fully symmetric field $\varphi_{\mu_{1} \ldots \mu_{s}}$ with $s>2$, that at the linearised level admits the gauge transformations (2.6) and satisfies the field equation (2.8).

Let us now present an alternative description of the free dynamics that will prove convenient to discuss interactions for these fields. As in the frame formulation of gravity one can substitute the fully symmetric Fronsdal field with a 1 -form $e_{\mu}{ }^{a_{1} \ldots a_{s-1}}$ [26]. In the standard frame-like approach this vielbein-like field is traceless and fully symmetric in its flat indices. However, due to the curved index, it carries a hooked $\{s-1,1\}$ component ${ }^{4}$ that is absent in the fully symmetric $\varphi_{\mu_{1} \ldots \mu_{s}}$. To eliminate it one resorts to a local Lorentz-like gauge transformation with a $\{s-1,1\}$-projected parameter, so that

$$
\delta e_{\mu}^{a_{1} \ldots a_{s-1}}=D_{\mu} \xi^{a_{1} \ldots a_{s-1}}+\bar{e}_{\mu, b} \Lambda^{b, a_{1} \ldots a_{s-1}},
$$

where $D_{\mu}$ is the Lorentz-covariant derivative while $\bar{e}_{\mu}{ }^{a}$ is the background vielbein. This leads to introduce a gauge connection $\omega_{\mu}{ }^{b, a_{1} \ldots a_{s-1}}$ for the new gauge transformation. It is the higher-spin analogue of the spin connection of gravity. The new field is traceless and $\{s-1,1\}$-projected in its flat indices. As in the gravity case, it must be an auxiliary field and it will be expressed in terms of $e_{\mu}{ }^{a_{1} \ldots a_{s-1}}$ and its first derivatives via suitable torsion-like constraints.

In conclusion, a spin- $s$ field freely propagating in a constant curvature background of arbitrary dimension can be described by the pair of one-forms

$$
e_{\mu}^{a_{1} \ldots a_{s-1}}, \quad \omega_{\mu}^{b, a_{1} \ldots a_{s-1}},
$$

which are irreducible Lorentz tensors in the flat indices. The Fronsdal formulation is recovered by eliminating the auxiliary field $\omega_{\mu}{ }^{b}, a_{1} \ldots a_{s-1}$ and considering the Lorentz-like invariant combination

$$
\varphi_{\mu_{1} \ldots \mu_{s}} \equiv \frac{1}{s} \bar{e}_{\left(\mu_{1}\right.}^{a_{1}} \ldots \bar{e}_{\mu_{s-1}}^{a_{s-1}} e_{\left.\mu_{s}\right) a_{1} \ldots a_{s-1}}
$$

with the gauge transformations induced by those of the vielbein-like potential. Notice that the tracelessness condition on the vielbein-like field $e_{\mu}{ }^{a_{1} \ldots a_{s-1}}$ induces the Fronsdal double trace constraints (2.4) on the metric-like field $\varphi_{\mu_{1} \ldots \mu_{s}}$. In [26] Vasiliev identified a first-order action for $e_{\mu}{ }^{a_{1} \ldots a_{s-1}}$ and $\omega_{\mu}{ }^{b, a_{1} \ldots a_{s-1}}$ describing the correct spin- $s$ free dynamics in a four-dimensional Minkowski background. In [27] he extended this result to constant curvature spaces and to arbitrary space-time dimensions. An important observation is that in general the resulting action is invariant under an enlarged set of gauge transformations. For instance, in a Minkowski background the free action is left invariant by the transformations

$$
\begin{aligned}
& \delta e_{\mu}^{{ }^{a_{1} \ldots a_{s-1}}}=\partial_{\mu} \xi^{a_{1} \ldots a_{s-1}}+\bar{e}_{\mu, b} \Lambda^{b, a_{1} \ldots a_{s-1}} \\
& \delta \omega_{\mu}^{b, a_{1} \ldots a_{s-1}}=\partial_{\mu} \Lambda^{b, a_{1} \ldots a_{s-1}}+\bar{e}_{\mu, c} \Theta^{b c, a_{1} \ldots a_{s-1}}
\end{aligned}
$$

\footnotetext{
${ }^{4}$ Here and in the following we classify tensors by representations of the permutation group acting on their indices. These are labelled by Young diagrams, that we identify by ordered lists of the lengths of their rows enclosed between braces.
} 
where $\Theta^{b_{1} b_{2}, a_{1} \ldots a_{s-1}}$ is an additional traceless and $\{s-1,2\}$-projected Stückelberg-like gauge parameter. Similar expressions hold in the AdS case, for which we refer the interested reader to the second reference of [27]. The appearance of a new gauge parameter calls for the introduction of an extra gauge connection. The procedure iterates until all gauge connections

$$
\omega_{\mu}^{b_{1} \ldots b_{t}, a_{1} \ldots a_{s-1}}, \quad 2 \leq t \leq s-1,
$$

are introduced. They are traceless and $\{s-1, t\}$-projected in their flat indices. They are usually called extra fields and they are necessary in order to rewrite the field equations in terms of curvatures, i.e. in terms of relations between gauge invariant objects. Even if extra fields do not enter the free action they do play a crucial role in Vasiliev's interacting theory [3]. However, in $D=3$ the gauge parameter $\Theta^{b_{1} b_{2}, a_{1} \ldots a_{s-1}}$ vanishes and also the extra fields do. In fact, for $O(n)$ groups the representations associated to Young diagrams with more than $n$ boxes in the first two columns vanish (see for instance [28], §10-6). As a result, in the following we shall ignore extra fields. Furthermore, restricting the attention to the proper orthogonal subgroups $O^{+}(n)$, the representations with $a$ boxes in the first column and those with $n-a$ boxes in the first column are equivalent (see again [28], $\S 10-6)$. In the three-dimensional context an example of this fact is the possibility to use the connection

$$
\omega_{\mu}^{a}=\frac{1}{2} \epsilon^{a b c} \omega_{\mu, b, c},
$$

rather than the usual spin connection $\omega_{\mu}^{a, b}$. The same is thus true for generic higher spins. In $D=3$ they can be described by the pair of gauge potentials

$$
e_{\mu}^{a_{1} \ldots a_{s-1}}, \quad \omega_{\mu}^{a_{1} \ldots a_{s-1}},
$$

sharing the same index structure. For more details on the frame-like formulation of the dynamics we refer to [3] and the references therein. In the next section, we shall take this last observation as a starting point for extending to higher spins the Chern-Simons reformulation of three-dimensional gravity.

\subsection{Chern-Simons formulation}

In presence of a negative cosmological constant three-dimensional Einstein gravity is equivalent to a Chern-Simons theory with gauge group $S O(2,2) \sim S L(2, \mathbb{R}) \times S L(2, \mathbb{R})$ $[13,14]$. A CS reformulation is available also for its supergravity extensions, with a gauge group which is the product of two supersymmetric extensions of $S L(2, \mathbb{R})$ [13]. In both cases the field equations are zero-curvature conditions and thus no local degrees of freedom are involved. As we discussed, in $D=3$ this property holds also for a gauge field $\varphi_{\mu_{1} \ldots \mu_{s}}$ satisfying the Fronsdal equation (2.8), and in fact in [15] Blencowe proposed an interacting theory for higher-spin fields in $D=3$ based on a CS action. In particular, he considered a gauge group which is the product of two-copies of an infinite-dimensional extension of $S L(2, \mathbb{R})$, thus mimicking the Fradkin-Vasiliev algebra driving higher-spin interactions in a four-dimensional AdS background [29]. However, as repeatedly stressed 
in the Introduction, in $D=3$ there is no need to consider an infinite tower of higher-spin fields in order to obtain consistent interactions. Therefore, in the following we shall review Blencowe's idea identifying the basic structures needed to couple any given spin- $s$ gauge field to gravity. However, in general this could require the simultaneous presence of other fields with different spin.

In order to reformulate Einstein gravity in $D=3$ as a CS theory, one defines linear combinations of dreibein and spin connection as ( $l$ denotes the AdS radius)

$$
\jmath_{\mu}{ }^{a}=\omega_{\mu}{ }^{a}+\frac{1}{l} e_{\mu}{ }^{a}, \quad \tilde{\jmath}_{\mu}{ }^{a}=\omega_{\mu}{ }^{a}-\frac{1}{l} e_{\mu}{ }^{a},
$$

and interprets $\jmath$ and $\tilde{\jmath}$ as $\operatorname{sl}(2, \mathbb{R})$ gauge potentials. In a similar fashion one defines the linear combinations

$$
t_{\mu}^{a_{1} \ldots a_{s-1}}=\left(\omega+\frac{e}{l}\right)_{\mu}^{a_{1} \ldots a_{s-1}}, \quad \tilde{t}_{\mu}^{a_{1} \ldots a_{s-1}}=\left(\omega-\frac{e}{l}\right)_{\mu}^{a_{1} \ldots a_{s-1}} .
$$

of the fields (2.16). One contracts them with some higher-spin generators $T_{a_{1} \ldots a_{s-1}}$, to be added to the $\operatorname{sl}(2, \mathbb{R})$ ones, and considers the one-forms

$$
\begin{aligned}
A & =\left(\jmath_{\mu}{ }^{a} J_{a}+t_{\mu}{ }^{a_{1} \ldots a_{s-1}} T_{a_{1} \ldots a_{s-1}}\right) d x^{\mu} \\
\widetilde{A} & =\left(\tilde{\jmath}_{\mu}{ }^{a} J_{a}+\tilde{t}_{\mu}{ }^{a_{1} \ldots a_{s-1}} T_{a_{1} \ldots a_{s-1}}\right) d x^{\mu}
\end{aligned}
$$

Since no local degrees of freedom should be involved, in $D=3$ it is natural to identify the equations of motion for a spin- $s$ gauge field coupled to gravity with flatness conditions for $A$ and $\widetilde{A}$. This leads, at the action level, to a CS theory. We shall now support this conclusion by checking that the resulting field equations reduce to the Fronsdal one (2.8) at the linearised level. To this end, we have to impose conditions on the higher-spin generators. First of all, since they are contracted with the potentials (2.18), they must transform as irreducible $s o(1,2) \sim \operatorname{sl}(2, \mathbb{R})$ tensors. Therefore, they must be symmetric and traceless in their indices (i.e. $T^{b}{ }_{b a_{3} \ldots a_{s-1}}=0$ ) and, as the $J_{a}$ satisfy

$$
\left[J_{a}, J_{b}\right]=\epsilon_{a b c} J^{c}
$$

they must satisfy

$$
\left[J_{a}, T_{b_{1} \ldots b_{s-1}}\right]=\epsilon^{m}{ }_{a\left(b_{1}\right.} T_{\left.b_{2} \ldots b_{s-1}\right) m}
$$

If the $J_{a}$ and the $T_{a_{1} \ldots a_{s-1}}$ generate a Lie algebra $\mathfrak{g}$ admitting a non-degenerate bilinear form (denoted in the following by tr) one can then consider the CS action

$$
S_{C S}[A]=\frac{k}{4 \pi} \int \operatorname{tr}\left(A \wedge d A+\frac{2}{3} A \wedge A \wedge A\right) .
$$

In [14] it was pointed out that the combination

$$
S=S_{C S}[A]-S_{C S}[\widetilde{A}]
$$


reduces to the Einstein-Hilbert action, up to boundary terms, when $A$ and $\widetilde{A}$ only contain the gravitational fields $\jmath$ and $\tilde{\jmath}$. In particular, with the conventional normalization

$$
\operatorname{tr}\left(J_{a} J_{b}\right)=\frac{1}{2} \eta_{a b}
$$

this identification leads to the relation

$$
k=\frac{l}{4 G},
$$

where $G$ is Newton's constant. As a result, eq. (2.23) provides the correct description of the gravitational sector, and we can check that the linearisation of its equations of motion also describes the free-propagation of a spin- $s$ field $\varphi_{\mu_{1} \ldots \mu_{s}}$ on an $A d S_{3}$ background. This ensures that the full interacting theory describes the coupling of $\varphi_{\mu_{1} \ldots \mu_{s}}$ to gravity.

To linearise the field equations derived from the action (2.23), one splits the gravitational dreibein and spin connection into background, $\bar{e}_{\mu}{ }^{a}$ and $\bar{\omega}_{\mu}{ }^{a}$, and fluctuations,

$$
e_{\mu}^{a}=\bar{e}_{\mu}^{a}+h_{\mu}^{a}, \quad \omega_{\mu}^{a}=\bar{\omega}_{\mu}^{a}+v_{\mu}^{a},
$$

and treats the higher-spin fields as fluctuations around trivial background values. Notice that the commutator of two higher-spin generators is not needed for the linearised field equations. Returning to the description in terms of the potentials (2.16), the commutators (2.20) and (2.21) imply that the spin-2 fluctuations satisfy

$$
\begin{aligned}
\mathscr{T}^{a} & \equiv D h^{a}+\epsilon^{a b c} \bar{e}_{b} \wedge v_{c}=0, \\
\mathscr{R}^{a} & \equiv D v^{a}+\frac{1}{l^{2}} \epsilon^{a b c} \bar{e}_{b} \wedge h_{c}=0,
\end{aligned}
$$

while the spin- $s$ fluctuations satisfy

$$
\begin{aligned}
& \mathscr{T}^{a_{1} \ldots a_{s-1}} \equiv D h^{a_{1} \ldots a_{s-1}}+\epsilon^{c d\left(a_{1} \mid\right.} \bar{e}_{c} \wedge v_{d}{ }^{\left.\mid a_{2} \ldots a_{s-1}\right)}=0, \\
& \mathscr{R}^{a_{1} \ldots a_{s-1}} \equiv D v^{a_{1} \ldots a_{s-1}}+\frac{1}{l^{2}} \epsilon^{c d\left(a_{1} \mid\right.} \bar{e}_{c} \wedge h_{d}{ }^{\left.\mid a_{2} \ldots a_{s-1}\right)}=0 .
\end{aligned}
$$

For brevity we omitted the form indices and we introduced the AdS covariant exterior derivative

$$
D f^{a_{1} \ldots a_{n}}=d f^{a_{1} \ldots a_{n}}+\epsilon^{c d\left(a_{1} \mid\right.} \bar{\omega}_{c} \wedge f_{d}^{\left.\mid a_{2} \ldots a_{n}\right)} .
$$

Notice that the field equations for the graviton are a particular case of those for a generic higher-spin gauge field freely propagating in an $A d S_{3}$ background. These field equations are left invariant by the transformations

$$
\begin{aligned}
& \left.\delta h^{a_{1} \ldots a_{s-1}}=D \xi^{a_{1} \ldots a_{s-1}}+\epsilon^{c d\left(a_{1} \mid\right.} \bar{e}_{c} \Lambda_{d} \mid a_{2} \ldots a_{s-1}\right) \\
& \delta v^{a_{1} \ldots a_{s-1}}=D \Lambda^{a_{1} \ldots a_{s-1}}+\frac{1}{l^{2}} \epsilon^{c d\left(a_{1} \mid\right.} \bar{e}_{c} \xi_{d} d^{\left.\mid a_{2} \ldots a_{s-1}\right)}
\end{aligned}
$$


since their gauge variation is proportional to the field equations for the background fields. This is the full set of gauge transformations of the linearised action, confirming the absence of extra fields in $D=3$.

We have thus identified the linearised field equations implied by the CS action (2.23) associated to any Lie algebra $\mathfrak{g} \oplus \mathfrak{g}$ with a semisimple $\mathfrak{g}$ generated by $J_{a}$ and $T_{a_{1} \ldots a_{s-1}}$ satisfying eqs. (2.20) and (2.21). We can now verify that they imply the Fronsdal equation (2.8) for the field $\varphi_{\mu_{1} \ldots \mu_{s}}$ of eq. (2.12), while the gauge transformations (2.30) imply the gauge transformations (2.6) for $\varphi_{\mu_{1} \ldots \mu_{s}}$. After this last step we shall eventually present a class of simple Lie algebras fitting into this scheme.

We start by noticing that the first of eqs. (2.28) is a generalisation of the torsion constraint of pure gravity and it can be used to express $v_{\mu}^{a_{1} \ldots a_{s-1}}$ in terms of $h_{\mu}{ }^{a_{1} \ldots a_{s-1}}$. In fact

$$
\epsilon^{\mu \nu \rho} \mathscr{T}_{\mu \nu}{ }^{a_{1} \ldots a_{s-1}}=0
$$

describes a square system of algebraic equations for the various components of $v_{\mu}{ }^{a_{1} \ldots a_{s-1}}$. This property holds only in $D=3$, while in higher space-time dimensions the mismatch between the number of equations and the number of components of $v$ is another evidence of the need for the extra Stückelberg-like gauge symmetry of eq. (2.13). Moreover, we can exhibit the general solution of eq. (2.31), thus proving that its determinant is different from zero when the background dreibein is invertible. It reads

$$
\begin{aligned}
& (s-1)^{2} v^{b, a_{1} \ldots a_{s-1}}=(s-3) \bar{e}^{b\left(a_{1} \mid\right.} v_{c}^{\left.c \mid a_{2} \ldots a_{s-2}\right)}-2 \bar{e}^{\left(a_{1} a_{2} \mid\right.} v_{c}^{\left.b c \mid a_{3} \ldots a_{s-2}\right)} \\
& \left.+(s-2) \epsilon^{b c d} \bar{e}_{c}^{\mu} \bar{e}^{\nu,\left(a_{1} \mid\right.} D_{[\mu} h_{\nu] d} d^{\left.\mid a_{2} \ldots a_{s-1}\right)}-\epsilon^{c d\left(a_{1} \mid\right.} \bar{e}_{c}^{\mu} \bar{e}^{\nu, b} D_{[\mu} h_{\nu] d} \mid a_{2} \ldots a_{s-1}\right) \\
& \left.-\epsilon^{c d\left(a_{1} \mid\right.} \bar{e}^{\mu}{ }_{c} \bar{e}^{\nu,\left|a_{2}\right|} D_{[\mu} h_{\nu] d} \mid a_{3} \ldots a_{s-1}\right) b
\end{aligned}
$$

where the mixed trace of $v$ is

$$
v_{b}^{b a_{1} \ldots a_{s-2}}=\frac{1}{2 s} \epsilon^{b c d} \bar{e}_{c}^{\mu} \bar{e}_{d}^{\nu} D_{[\mu} h_{\nu] b}{ }^{a_{1} \ldots a_{s-2}} .
$$

In eqs. (2.32) and (2.33) the square brackets denote the antisymmetrisation of the indices they enclose, again with unit overall normalisation. As in the gravity case, the invertibility of the background dreibein plays a crucial role in the identification of the relation (2.32).

Substituting the solution of eq. (2.31) in the linearised CS action one then obtains a second-order action depending on

$$
h_{\mu_{1}, \mu_{2} \ldots \mu_{s}}=\bar{e}_{\mu_{2}}^{a_{1}} \ldots \bar{e}_{\mu_{s}}^{a_{s-1}} h_{\mu_{1}, a_{1} \ldots a_{s-1}} .
$$

But acting with the Lorentz-like gauge transformation (2.30) generated by $\Lambda^{a_{1} \ldots a_{s-1}}$ it is possible to eliminate the $\{s, 1\}$-component carried by this combination. As a result, the action eventually depends only on the field (2.12), whose gauge transformations can be deduced by acting with (2.30) in eq. (2.12). In performing this substitution one can also eliminate the background spin connection appearing in the result by using the vielbein postulate

$$
\partial_{\mu} e_{\nu}^{a}+\epsilon_{b c}^{a} \omega_{\mu}^{b} e_{\nu}^{c}-\Gamma_{\mu \nu}^{\lambda} e_{\lambda}^{a}=0 .
$$


The $A d S_{3}$ Christoffel symbols so introduced enable one to cast the gauge transformation in the form

$$
\delta \varphi_{\mu_{1} \ldots \mu_{s}}=\nabla_{\left(\mu_{1}\right.} \xi_{\left.\mu_{2} \ldots \mu_{s}\right)}
$$

with

$$
\xi_{\mu_{1} \ldots \mu_{s-1}}=\bar{e}_{\mu_{1}}^{a_{1}} \ldots \bar{e}_{\mu_{s-1}}^{a_{s-1}} \xi_{a_{1} \ldots a_{s-1}} .
$$

Moreover, the tracelessness conditions on $\xi^{a_{1} \ldots a_{s-1}}$ and $h_{\mu}{ }^{{ }^{1} \ldots a_{s-1}}$ induce the Fronsdal constraints (2.3) and (2.4) on $\xi_{\mu_{1} \ldots \mu_{s-1}}$ and $\varphi_{\mu_{1} \ldots \mu_{s}}$. The resulting action thus coincides with the Fronsdal one (2.9), since its structure is fixed by the requirement of gauge invariance under the transformations (2.36).

To summarise, we have reduced the problem of finding a consistent gravitational coupling for a spin- $s$ field to the problem of finding a semisimple Lie algebra whose generators can be split in $J_{a}$ and $T_{a_{1} \ldots a_{s}}$ satisfying eqs. (2.20) and (2.21). The Jacobi identities and the trace constraints could impose strong restrictions and a priori it could be necessary to simultaneously consider more higher-spin fields to fulfil them. ${ }^{5}$ A direct constructive approach could then end up in a rather non-trivial task, but in $[30,16]$ it was shown how to describe a generic $\operatorname{sl}(n)$ algebra in terms of generators $T_{a_{1} \ldots a_{s-1}}$ with $2 \leq s \leq n$, traceless and fully symmetric in the indices they carry. This result provides a first interesting class of examples fitting into the previous discussion.

The starting point is the observation that any symmetrised product of $s l(2, \mathbb{R})$ generators of the form

$$
T_{a_{1} \ldots a_{s-1}} \sim J_{\left(a_{1}\right.} \ldots J_{\left.a_{s-1}\right)}
$$

satisfies the commutator (2.21) with $J_{a}$. Their traceless projections thus satisfy the properties that identify possible higher-spin generators, but in general the commutator between generators with spins $s_{1}$ and $s_{2}$ produces a new $T$ with spin

$$
\left|s_{1}-s_{2}\right|+1 \leq s_{3} \leq s_{1}+s_{2}-1
$$

thus preventing the realisation of a finite-dimensional algebra. However, if one considers a $n$-dimensional representation for the $J_{a}$, the $T_{a_{1} \ldots a_{s-1}}$ are $n \times n$ matrices. The tracelessness condition in the $a_{n}$ indices then implies that they are traceless matrices. Furthermore, the whole set of matrices generated by the combinations (2.38) with $s \leq n$ contains $n^{2}-1$ independent elements $[30,16]$. Therefore, even if this argument does not suffice to identify the precise form of the commutators between higher-spin generators, it ensures that the first $n-1$ products (2.38) generate the $\operatorname{sl}(n)$ algebra when one deals with a $n$-dimensional representation of $\operatorname{sl}(2, \mathbb{R})$. The particular real form that one realises depends on the choice of the normalisation of eq. (2.38), as we shall see in the next section in the spin-3 example. This presentation of $s l(n)$ implies that a $S L(n) \times S L(n)$ CS theory can be interpreted as describing the coupling of a tower of fields of increasing spin $2,3, \ldots, n$, where each value of the spin appears only once. In the limit $n \rightarrow \infty$ the present construction leads

\footnotetext{
${ }^{5}$ In principle even the choice $\left[T_{a_{1} \ldots a_{s-1}}, T_{b_{1} \ldots b_{s-1}}\right]=0$ would be consistent, but the resulting algebra actually describes only the free-propagation of higher-spin gauge fields.
} 
to the higher-spin gauge theory based on the algebra of area-preserving diffeomorphisms on a two-dimensional hyperboloid, which was discussed in detail in [16]. In the following we shall mainly examine the properties of this class of higher-spin gauge theories - and in particular of its simplest example describing the coupling of a spin-3 field to gravity confining to Section 5 some comments on more general alternatives.

\subsection{The spin-3 example}

In the spin-3 case eqs. (2.20) and (2.21) allow for the introduction of a non-trivial commutator between the higher-spin generators $T_{a b}$, which is uniquely fixed by the Jacobi identity up to a normalisation constant $\sigma$. The resulting non-Abelian Lie algebra is

$$
\begin{aligned}
& {\left[J_{a}, J_{b}\right]=\epsilon_{a b c} J^{c},} \\
& {\left[J_{a}, T_{b c}\right]=\epsilon_{a(b}^{m} T_{c) m},} \\
& {\left[T_{a b}, T_{c d}\right]=\sigma\left(\eta_{a(c} \epsilon_{d) b m}+\eta_{b(c} \epsilon_{d) a m}\right) J^{m},}
\end{aligned}
$$

where the $T_{a b}$ are traceless and symmetric in $a, b$. Notice that the right-hand side of eq. $(2.40 \mathrm{~b})$ is traceless in the indices $b, c$, while the right-hand side of eq. (2.40c) is traceless in $a, b$ and $c, d$. Imposing $T_{a}^{a}=0$ is thus consistent. The algebra (2.40) has the quadratic Casimir

$$
C=J_{a} J^{a}-\frac{1}{2 \sigma} T_{a b} T^{a b}
$$

that enables to define a non-degenerate bilinear form on it. Actually, one can show that $(2.40)$ is isomorphic to $\operatorname{sl}(3, \mathbb{C})$, and thus matches the corresponding algebra obtained

from the construction discussed at the end of the previous section. One can build its fundamental representation by defining the $T_{a b}$ generators as

$$
T_{a b}=\sqrt{-\sigma}\left(J_{(a} J_{b)}-\frac{2}{3} \eta_{a b} J_{c} J^{c}\right)
$$

where the $J_{a}$ are the $\operatorname{sl}(2, \mathbb{R})$ generators in the 3 -dimensional representation. The sign of $\sigma$ selects one of the two non-compact real forms of $\operatorname{sl}(3, \mathbb{C})$. In fact, the non-compact subalgebra $\operatorname{sl}(2, \mathbb{R})$ rules out the compact real form, while a real rescaling of the generators $T_{a b}$ can modify the absolute value of $\sigma$ but not its sign. In particular, $\sigma>0$ corresponds to $s u(1,2)$, while $\sigma<0$ corresponds to $\operatorname{sl}(3, \mathbb{R})$. Notice that the analogy with the spin-2 case is not sufficient to single out one of the two real forms of $\operatorname{sl}(3, \mathbb{C})$. In fact, $\operatorname{sl}(2, \mathbb{C})$ admits only a single non-compact real form since $\operatorname{su}(1,1) \sim \operatorname{sl}(2, \mathbb{R})$.

Since the algebra $(2.40)$ is isomorphic to $\operatorname{sl}(3, \mathbb{C})$, it is convenient to rewrite it in a more standard basis where one does not have to deal with the trace constraints on the 
generators. In particular, in the following we shall use the basis

$$
\begin{aligned}
& {\left[L_{i}, L_{j}\right]=(i-j) L_{i+j},} \\
& {\left[L_{i}, W_{m}\right]=(2 i-m) W_{i+m},} \\
& {\left[W_{m}, W_{n}\right]=\frac{\sigma}{3}(m-n)\left(2 m^{2}+2 n^{2}-m n-8\right) L_{m+n},}
\end{aligned}
$$

where $-1 \leq i, j \leq 1$ and $-2 \leq m, n \leq 2$. It can be related to the previous one via the isomorphism

$$
J_{0}=\frac{1}{2}\left(L_{1}+L_{-1}\right), \quad J_{1}=\frac{1}{2}\left(L_{1}-L_{-1}\right), \quad J_{2}=L_{0},
$$

that for the spin-3 generators reads

$$
\begin{aligned}
T_{00} & =\frac{1}{4}\left(W_{2}+W_{-2}+2 W_{0}\right), & T_{01} & =\frac{1}{4}\left(W_{2}-W_{-2}\right), \\
T_{11} & =\frac{1}{4}\left(W_{2}+W_{-2}-2 W_{0}\right), & T_{02} & =\frac{1}{2}\left(W_{1}+W_{-1}\right), \\
T_{22} & =W_{0}, & T_{12} & =\frac{1}{2}\left(W_{1}-W_{-1}\right) .
\end{aligned}
$$

Notice that eq. (2.45) makes manifest the traceless condition

$$
-T_{00}+T_{11}+T_{22}=0 .
$$

The $W_{m}$ generators thus provide a convenient parameterisation of the independent components of the $T_{a b}$ generators. The elimination of the trace constraints on the generators leads to important technical simplifications. In this respect, our choice represent an alternative to the spinorial notation adopted by Vasiliev [27] and Blencowe [15] to the same end.

In conclusion, one can describe the coupling of a spin-3 gauge field to AdS gravity in $D=3$ via the CS action (2.23) associated to the direct sum of two copies of the algebra (2.40). Since $\sigma$ appears only in the commutator between two spin-3 generators it does not affect the linearised field equations. Therefore, it is possible to consider any direct sum of the two non-compact real forms of $\operatorname{sl}(3, \mathbb{C})$. However, choosing the direct sum of the same real algebra entails a qualitative difference with respect to the choice of two different real forms. The distinction emerges when one performs the change of basis that induces the rewriting of the field equations in terms of the potentials (2.16):

$$
\begin{array}{lll}
M_{a}=J_{a}+\widetilde{J}_{a}, & M_{a b}=T_{a b}+\widetilde{T}_{a b}, \\
P_{a}=\frac{1}{l}\left(J_{a}-\widetilde{J}_{a}\right), & P_{a b}=\frac{1}{l}\left(T_{a b}-\widetilde{T}_{a b}\right),
\end{array}
$$

where the tilde distinguishes the two copies of $\operatorname{sl}(3, \mathbb{C})$. The commutators between the $P_{a}$ and the $M_{a}$ are not affected by the choice of $\sigma$ and $\tilde{\sigma}$ and one recovers the usual presentation of the $o(2,2)$ algebra:

$$
\left[M_{a}, M_{b}\right]=\epsilon_{a b c} M^{c}, \quad\left[M_{a}, P_{b}\right]=\epsilon_{a b c} P^{c}, \quad\left[P_{a}, P_{b}\right]=\frac{1}{l^{2}} \epsilon_{a b c} M^{c} .
$$


In a similar fashion, also the commutators mixing spin-2 and spin-3 generators are independent on the choice of $\sigma$ and $\tilde{\sigma}$ :

$$
\begin{array}{ll}
{\left[M_{a}, M_{b c}\right]=\epsilon_{a(b}^{m} M_{c) m},} & {\left[M_{a}, P_{b c}\right]=\epsilon_{a(b}^{m} P_{c) m},} \\
{\left[P_{a}, M_{b c}\right]=\epsilon_{a(b}^{m} P_{c) m},} & {\left[P_{a}, P_{b c}\right]=\frac{1}{l^{2}} \epsilon_{a(b}^{m} M_{c) m} .}
\end{array}
$$

On the other hand, the structure of the remaining commutators depends on $\sigma$ and $\tilde{\sigma}$ as

$$
\begin{aligned}
& {\left[M_{a b}, M_{c d}\right]=\frac{1}{2}\left(\eta_{a(c} \epsilon_{d) b m}+\eta_{b(c} \epsilon_{d) a m}\right)\left((\sigma+\tilde{\sigma}) M^{m}+l(\sigma-\tilde{\sigma}) P^{m}\right),} \\
& {\left[M_{a b}, P_{c d}\right]=\frac{1}{2}\left(\eta_{a(c} \epsilon_{d) b m}+\eta_{b(c} \epsilon_{d) a m}\right)\left((\sigma+\tilde{\sigma}) P^{m}+\frac{1}{l}(\sigma-\tilde{\sigma}) M^{m}\right),} \\
& {\left[P_{a b}, P_{c d}\right]=\frac{1}{2}\left(\eta_{a(c} \epsilon_{d) b m}+\eta_{b(c} \epsilon_{d) a m}\right)\left(\frac{1}{l^{2}}(\sigma+\tilde{\sigma}) M^{m}+\frac{1}{l}(\sigma-\tilde{\sigma}) P^{m}\right) .}
\end{aligned}
$$

By a suitable real rescaling of the generators the absolute values of $\sigma$ and $\tilde{\sigma}$ can be equated, but one can contract the full algebra with a $l \rightarrow \infty$ limit only if they have the same sign. A similar obstruction was recognised in [31] for the supergravity case, where one can consider two possible supergroups $O S p_{ \pm}(\mathcal{N} \mid 2, \mathbb{R})$ depending on the overall sign of the anticommutator of the fermionic generators. In that case, to obtain a well defined Poincaré limit one has to choose two supergroups with opposite sign. At any rate, both choices could well be acceptable. For instance, in $D=4$ Vasiliev's theory for interacting higher-spin fields does not admit a flat-space limit [3].

We can now close this section by presenting the full non-linear action and the field equations in terms of the potentials (2.16). This makes the interpretation of the CS theory as the coupling of a spin-3 field to gravity more transparent. For simplicity we shall focus on $\sigma=\tilde{\sigma}$. In terms of the vielbein-like and of the spin-connection like fields the action $(2.23)$ reads

$$
\begin{aligned}
& S=\frac{1}{8 \pi G} \int\left\{e^{a} \wedge\left(d \omega_{a}+\frac{1}{2} \epsilon_{a b c} \omega^{b} \wedge \omega^{c}-2 \sigma \epsilon_{a b c} \omega^{b d} \wedge \omega_{d}^{c}\right)\right. \\
& \left.-2 \sigma e^{a b} \wedge\left(d \omega_{a b}+\epsilon_{c d(a \mid} \omega^{c} \wedge \omega_{\mid b)}{ }^{d}\right)+\frac{1}{6 l^{2}} \epsilon_{a b c}\left(e^{a} \wedge e^{b} \wedge e^{c}-12 \sigma e^{a} \wedge e^{b d} \wedge e_{d}^{c}\right)\right\} .
\end{aligned}
$$

Recall that for $\sigma>0$ the gauge group is $S U(1,2) \times S U(1,2)$ while for $\sigma<0$ it is $S L(3, \mathbb{R}) \times S L(3, \mathbb{R})$. The equations of motion for the gravitational fields are

$$
\begin{aligned}
\mathscr{T}^{a} & \equiv d e^{a}+\epsilon^{a b c} \omega_{b} \wedge e_{c}-4 \sigma \epsilon^{a b c} e_{b d} \wedge \omega_{c}{ }^{d}=0 \\
\mathscr{R}^{a} & \equiv d \omega^{a}+\frac{1}{2} \epsilon^{a b c}\left(\omega_{b} \wedge \omega_{c}+\frac{e_{b} \wedge e_{c}}{l^{2}}\right)-2 \sigma \epsilon^{a b c}\left(\omega_{b d} \wedge \omega_{c}{ }^{d}+\frac{e_{b d} \wedge e_{c}{ }^{d}}{l^{2}}\right)=0 .
\end{aligned}
$$

Notice that the spin-3 fields provide a contribution to the torsion equation analogue to that appearing in $\mathcal{N}=1$ supergravity $[13,31]$. Indeed, the structure of the algebra $(2.40)$ 
is very close to that of the $O S p_{ \pm}(1 \mid 2, \mathbb{R})$ superalgebra, since the commutator of two spin-3 generators is proportional to a spin- 2 generator. The field equations for the spin-3 fields are

$$
\begin{aligned}
& \mathscr{T}^{a b} \equiv d e^{a b}+\epsilon^{c d(a \mid} \omega_{c} \wedge e_{d}^{\mid b)}+\epsilon^{c d(a \mid} e_{c} \wedge \omega_{d}^{\mid b)}=0 \\
& \mathscr{R}^{a b} \equiv d \omega^{a b}+\epsilon^{c d(a \mid} \omega_{c} \wedge \omega_{d}^{\mid b)}+\frac{1}{l^{2}} \epsilon^{c d(a \mid} e_{c} \wedge e_{d}^{\mid b)}=0 .
\end{aligned}
$$

The coupling also deforms the gauge transformations. Besides the usual gauge transformations, the spin-2 fields acquire new gauge transformations proportional to the spin-3 gauge parameters $\xi^{a b}$ and $\Lambda^{a b}$ :

$$
\begin{aligned}
& \delta e^{a}=-4 \sigma \epsilon^{a b c} \omega_{b d} \xi_{c}^{d}-4 \sigma \epsilon^{a b c} e_{b d} \Lambda_{c}{ }^{d} \\
& \delta \omega^{a}=-4 \sigma \epsilon^{a b c} \omega_{b d} \Lambda_{c}{ }^{d}-4 \frac{\sigma}{l^{2}} \epsilon^{a b c} e_{b d} \xi_{c}{ }^{d}
\end{aligned}
$$

Similarly, the spin-3 fields also transforms under spin-2 gauge transformations. Their most general gauge transformations read

$$
\begin{aligned}
& \left.\left.\delta e^{a b}=d \xi^{a b}+\epsilon^{c d(a \mid} \omega_{c} \xi_{d} \mid b\right)+\epsilon^{c d(a \mid} e_{c} \Lambda_{d} \mid b\right)+\epsilon^{c d(a} \omega^{b)}{ }_{c} \xi_{d}+\epsilon^{c d(a} e^{b)}{ }_{c} \Lambda_{d} \\
& \left.\left.\delta \omega^{a b}=d \Lambda^{a b}+\epsilon^{c d(a \mid} \omega_{c} \Lambda_{d} \mid b\right)+\frac{1}{l^{2}} \epsilon^{c d(a \mid} e_{c} \xi_{d} \mid b\right)+\epsilon^{c d(a} \omega^{b)}{ }_{c} \Lambda_{d}+\frac{1}{l^{2}} \epsilon^{c d(a} e^{b)}{ }_{c} \xi_{d}
\end{aligned}
$$

\section{Review of Chern-Simons theory with boundary}

This section is a review of well known facts on CS theory in $D=3$, which we include for completeness of the presentation and to fix the notation. We largely follow the expositions in $[32,33,34]$.

Consider a generic Chern-Simons theory with gauge group $G$ (generated by the Lie algebra $\mathfrak{g}$ ) on a space $\mathcal{M}=\mathbb{R} \times \Sigma$, where $\Sigma$ is a two-dimensional manifold with boundary $\partial \Sigma \cong S^{1}$. On manifolds with boundary the CS action (2.22) in general is neither differentiable nor gauge invariant. In fact, when one varies it, one obtains the boundary contribution

$$
\left.\delta S_{C S}\right|_{\text {boundary }}=-\frac{k}{4 \pi} \int_{\mathbb{R} \times S^{1}} \operatorname{tr}(A \wedge \delta A) .
$$

In order to have a well defined action principle one either has to add boundary terms to the action (2.22) or to impose suitable boundary conditions on fields. As we shall see, the choice of boundary conditions will play a crucial role in our higher-spin setup. The presence of a timelike boundary affects the phase space of the theory. In general it becomes infinite dimensional, and there are infinitely many global charges satisfying an algebra that depends on the choice of boundary conditions. To find the algebra of global charges we follow the method of Regge and Teitelboim [35] as applied to the CS theory in $[32,33]$. 
The first step is to rewrite the CS action using a $(2+1)$-decomposition of the gauge field,

$$
A=A_{0} d t+A_{i} d x^{i}
$$

The action then reads

$$
S_{C S}=\frac{k}{4 \pi} \int_{\mathcal{M}} d t \wedge d x^{i} \wedge d x^{j} \operatorname{tr}\left(A_{0} F_{i j}-A_{i} \dot{A}_{j}\right)+\frac{k}{4 \pi} \int_{\mathbb{R} \times S^{1}} d t \wedge d x^{i} \operatorname{tr}\left(A_{0} A_{i}\right)
$$

This action has $2 N$ dynamical fields $A_{i}$ (where $N$ is the dimension of the gauge group $G$ ) and $N$ Lagrange multipliers $A_{0}$. The field equations of the Lagrange multipliers provide first class constraints which generate gauge transformations. The equal-time Poisson bracket of two differentiable phase-space functionals $F\left[A_{i}\right]$ and $H\left[A_{i}\right]$ is defined by

$$
\{F, H\}=\frac{2 \pi}{k} \int_{\Sigma} d x^{i} \wedge d x^{j} \operatorname{tr}\left(\frac{\delta F}{\delta A_{i}(x)} \frac{\delta H}{\delta A_{j}(x)}\right) .
$$

One defines the smeared generators of gauge transformations

$$
G(\Lambda)=\frac{k}{4 \pi} \int_{\Sigma} d x^{i} \wedge d x^{j} \operatorname{tr}\left(\Lambda F_{i j}\right)+Q(\Lambda)
$$

where $Q(\Lambda)$ is a boundary term whose role is to cancel the surface term that arises if one writes the variation of the first term in eq. (3.5) in terms of $\delta A_{i}$ rather than its derivatives [36].

True (proper) gauge transformations are those for which the surface term vanishes. If the gauge parameter $\Lambda$ is independent of the fields, the boundary term takes the form

$$
Q(\Lambda)=-\frac{k}{2 \pi} \int_{\partial \Sigma} d x^{i} \operatorname{tr}\left(\Lambda A_{i}\right)
$$

This leads to the Poisson algebra

$$
\{G(\Lambda), G(\Gamma)\}=G([\Lambda, \Gamma])+\frac{k}{2 \pi} \int_{\partial \Sigma} d x^{i} \operatorname{tr}\left(\Lambda \partial_{i} \Gamma\right)
$$

where the central extension crucially rests on the presence of the surface term $Q(\Lambda)$ in the definition of the smeared generator. Notice that the boundary contribution $Q(\Lambda)$ does not vanish when the constraints $F_{i j}=0$ are imposed. The transformations generated by a $G(\Lambda)$ with $\Lambda$ such that $Q(\Lambda)$ is non-zero, are not true gauge transformations, but rather global symmetries which transform physically inequivalent configurations into each other. This is also the origin of the infinitely many boundary degrees of freedom.

After gauge fixing and solving the constraints, the $Q(\Lambda)$ define the global charges of the CS theory. They generate global symmetries by acting on a generic phase-space functional $F$ as

$$
\delta_{\Lambda} F=\{Q(\Lambda), F\}
$$


and they satisfy the same algebra as the $G(\Lambda)$, i.e.

$$
\{Q(\Lambda), Q(\Gamma)\}=Q([\Lambda, \Gamma])+\frac{k}{2 \pi} \int_{\partial \Sigma} d x^{i} \operatorname{tr}\left(\Lambda \partial_{i} \Gamma\right),
$$

but now the brackets are Dirac brackets on the reduced phase space.

We now present a set of boundary conditions which ensure the differentiability of the CS action and a gauge-fixing procedure that will play a crucial role in the following. To select the boundary conditions it is convenient to introduce light cone coordinates $x^{ \pm}=\frac{t}{l} \pm \theta$, where $t$ parameterises the time direction while $\theta$ parameterises the circle at the boundary. Eq. (3.1) becomes

$$
\left.\delta S_{C S}\right|_{\text {boundary }}=-\frac{k}{4 \pi} \int_{\mathbb{R} \times S^{1}} d x^{+} d x^{-} \operatorname{tr}\left(A_{+} \delta A_{-}-A_{-} \delta A_{+}\right) .
$$

This vanishes if we impose

$$
A_{-}=0 \quad \text { at the boundary. }
$$

We shall later see that this choice can also be motivated from the gravity description: for instance, all black hole solutions and whatever is generated from them by the action of asymptotic Killing vectors/tensors satisfy this condition.

Let us now assume that the constant time slices $\Sigma$ have the topology of a disc, which we parameterise by a radial coordinate $\rho$ and the previous angle variable $\theta$. To fix the gauge, we choose a function $b(\rho)$ with values in the group $G$ and we set

$$
A_{\rho}=b^{-1}(\rho) \partial_{\rho} b(\rho) \text {. }
$$

This choice is always possible. Assume we start with a gauge field $A^{\prime}$, and we want to perform a gauge transformation $U$ to bring it to the form (3.12),

$$
U^{-1} A_{\rho}^{\prime} U+U^{-1} \partial_{\rho} U=b^{-1} \partial_{\rho} b .
$$

We write $U=U^{\prime} b$, and from (3.13) we obtain

$$
\partial_{\rho} U^{\prime}=-A_{\rho}^{\prime} U^{\prime}
$$

which can be solved by a path-ordered exponential,

$$
U=\mathcal{P} e^{-\int^{\rho} A_{\rho}^{\prime} d \rho^{\prime}} U_{0} b .
$$

$U_{0}$ is a constant of integration (independent of $\rho$ ) and is chosen such that $U=1$ at the boundary. This leaves the boundary condition (3.11) untouched. Therefore, $U$ is an allowed gauge transformation also in the theory with boundary, and the gauge choice (3.12) is always possible.

From the constraint $F_{\rho \theta}=0$ we then find

$$
\partial_{\rho} A_{\theta}+\left[A_{\rho}, A_{\theta}\right]=0
$$


which is solved by

$$
A_{\theta}(t, \rho, \theta)=b^{-1}(\rho) a(t, \theta) b(\rho) .
$$

The $\rho$-dependence of the Lagrange multiplier $A_{0}$ is determined by the equation of motion for the gauge-fixed (and therefore constant) $A_{\rho}$,

$$
\partial_{\rho} A_{0}+\left[A_{\rho}, A_{0}\right]=0
$$

The boundary condition $A_{-}=\frac{1}{2}\left(A_{0}-A_{\theta}\right)=0$ then forces $A_{0}$ to coincide with $A_{\theta}$ everywhere; in other words on shell

$$
A_{-} \equiv 0
$$

everywhere, not only at the boundary. Therefore, the phase space is parameterised by $a(t, \theta)$. The gauge choice (3.12) is preserved by gauge transformations whose parameters are of the form

$$
\Lambda(t, \rho, \theta)=b^{-1}(\rho) \lambda(t, \theta) b(\rho) \quad \Rightarrow \quad \delta A_{\rho}=0,
$$

but the boundary condition (3.11) implies that they generate global symmetries. In fact, an arbitrary time dependence for $\lambda(t, \theta)$ is not compatible with (3.11):

$$
\delta A_{-}=0 \quad \Rightarrow \quad \partial_{-} \lambda=0
$$

This is also the condition on the gauge parameter under which the CS action is gauge invariant. Similarly, the on-shell condition on $a(t, \theta)$ is that it depends only on $x^{+}$. This confirms the absence of an arbitrary time dependence and therefore proves that (3.12) is not only admissible, but also completely fixes the gauge freedom.

The Poisson structure on the reduced phase space can be obtained by inserting in eq. (3.9) the gauge-fixed expression for the charges (3.6),

$$
Q(\lambda)=-\frac{k}{2 \pi} \int d \theta \operatorname{tr}(\lambda(\theta) a(\theta))
$$

Expanding $a(\theta)$ in a basis $\left\{\mathcal{T}_{A}\right\}$ of the Lie algebra, $a=a^{A} \mathcal{T}_{A}$, one obtains the affine Lie algebra

$$
\left\{a^{A}(\theta), a^{B}\left(\theta^{\prime}\right)\right\}=-\frac{2 \pi}{k}\left(\delta\left(\theta-\theta^{\prime}\right) f_{C}^{A B} a^{C}(\theta)-\delta^{\prime}\left(\theta-\theta^{\prime}\right) \gamma^{A B}\right)
$$

where $f_{A B}{ }^{C}$ are the structure constant of $\mathfrak{g}$ and $\gamma^{A B}$ is the inverse of the Killing metric $\gamma_{A B}$. The Killing metric and its inverse are used to lower and raise the indices of the structure constants. Decomposing $a(\theta)$ into Fourier modes,

$$
a^{A}(\theta)=\frac{1}{k} \sum_{p \in \mathbb{Z}} a_{p}^{A} e^{-i p \theta},
$$

leads to

$$
\left\{a_{p}^{A}, a_{q}^{B}\right\}=-f^{A B}{ }_{C} a_{p+q}^{C}+i p k \gamma^{A B} \delta_{p+q, 0}
$$




\section{Asymptotic symmetries}

In this section we analyse the asymptotic symmetries of gravity coupled to a spin-3 field in backgrounds which are asymptotically AdS. We work in the frame-like formulation as a CS theory. First we motivate the boundary conditions we have to impose in the CS theory by reconsidering those of pure gravity, and then we define when a configuration is asymptotically $A d S$ in our higher-spin context. This leads to the identification of the Poisson structure on this solution space. The Poisson algebra structure we find is that of a classical centrally extended $\mathcal{W}_{3} \otimes \mathcal{W}_{3}$ algebra. Finally, we discuss the implications of our boundary conditions in the metric-like formulation.

\subsection{Boundary conditions}

As anticipated in Section 3, the boundary condition (3.11) emerges naturally when describing asymptotically Anti-de Sitter solutions of the field equations. On the other hand, it is not sufficient to fully characterise this class of solutions, and it has to be supplemented by further boundary conditions. We shall now confirm the role of (3.11) and identify the extra requirements by a close scrutiny at the properties of the asymptotically AdS solutions of Einstein gravity. In fact, any solution of the field equations of pure gravity can be embedded in any CS higher-spin extension. It simply corresponds to a solution where all higher-spin fields vanish. ${ }^{6}$ Therefore, these pure-gravity backgrounds provide a subset of the space of asymptotically AdS solutions we are going to characterise.

In [33] it was pointed out that the metric

$$
d s^{2}=l^{2}\left\{d \rho^{2}-\frac{8 \pi G}{l}\left(\mathcal{L}\left(d x^{+}\right)^{2}+\widetilde{\mathcal{L}}\left(d x^{-}\right)^{2}\right)-\left(e^{2 \rho}+\frac{64 \pi^{2} G^{2}}{l^{2}} \mathcal{L} \widetilde{\mathcal{L}} e^{-2 \rho}\right) d x^{+} d x^{-}\right\}
$$

is an exact solution of Einstein's equations for any $\mathcal{L}=\mathcal{L}\left(x^{+}\right)$and $\widetilde{\mathcal{L}}=\widetilde{\mathcal{L}}\left(x^{-}\right)$. Moreover, it parameterises the whole space of asymptotically $(\rho \rightarrow \infty)$ AdS solutions with a flat boundary metric. ${ }^{7}$ For instance, if we set the two functions $\mathcal{L}$ and $\widetilde{\mathcal{L}}$ to the constant values

$$
\mathcal{L}_{0}=-\frac{1}{4 \pi}(M l-J), \quad \widetilde{\mathcal{L}}_{0}=-\frac{1}{4 \pi}(M l+J),
$$

eq. (4.1) is the BTZ solution with mass $M>0$ and angular momentum $|J| \leq M l$. For $8 G M=-1$ and $J=0$ it is the $A d S_{3}$ solution. The change of coordinates casting the BTZ metric (4.1) in the usual form in terms of lapse and shift functions can be found in [33].

\footnotetext{
${ }^{6}$ The embedding of BTZ solutions into three-dimensional higher-spin gauge theories was already considered in [37] as a playground for the study of exact solutions in this context.

${ }^{7}$ This is, in fact, a special case of the general result [38] that in three dimensions the Fefferman-Graham expansion [39] terminates after the third term.
} 
Introducing $b(\rho)=e^{\rho L_{0}}$ the metric (4.1) can be described by the connections

$$
\begin{aligned}
& A=b^{-1}\left(L_{1}+\frac{2 \pi}{k} \mathcal{L}\left(x^{+}\right) L_{-1}\right) b d x^{+}+b^{-1} \partial_{\rho} b d \rho, \\
& \widetilde{A}=-b\left(\frac{2 \pi}{k} \widetilde{\mathcal{L}}\left(x^{-}\right) L_{1}+L_{-1}\right) b^{-1} d x^{-}+b \partial_{\rho} b^{-1} d \rho,
\end{aligned}
$$

that are related to the dreibein and the spin connection through eqs. (2.17) and (2.19). Notice that we exploited the relation (2.25) between the level of the CS action and the AdS radius. We also resorted to the basis (2.43) that will prove particularly convenient when we shall extend the discussion to the whole $S L(3) \times S L(3)$ CS theory.

The connections (4.3), which translate the Brown-Henneaux boundary conditions into the frame formalism, were introduced in [40] and it was pointed out in [33] that they provide an exact solution of the Einstein equations. Solutions parameterised by different functions $\mathcal{L}$ and $\widetilde{\mathcal{L}}$ cannot be related by proper gauge transformations, and thus they are physically inequivalent. This characterisation of the space of asymptotically AdS solutions was extended to supergravity theories with one or several spin-3/2 fields in [41]. Our generalisation consists in including a spin-3 field which is coupled to gravity. Further possible generalisation with several fields with integer and/or half-integer spins $>2$ based on higher rank groups and supergroups should be straightforward, but we will not explicitly consider them here; see however the discussion in Section 5.

Notice that the connection $A$ of eq. (4.3) satisfies the gauge choice (3.12) and the condition (3.19) in the whole space and $\widetilde{A}$ satisfies analogous conditions. We shall now show that these properties continue to hold on the wider space of solutions which is obtained by acting on a generic pure-gravity background of the form (4.3) with the isometries of the $A d S_{3}$ solution. As such, they can be considered as crucial ingredients in the characterisation of generic asymptotically AdS solutions even in the full $S L(3) \times S L(3) \mathrm{CS}$ theory.

To prove this statement, notice that in the AdS case, for which $\frac{2 \pi}{k} \mathcal{L}=\frac{2 \pi}{k} \widetilde{\mathcal{L}}=\frac{1}{4}$, eq. (4.3) can be written as

$$
A=g^{-1} d g, \quad \widetilde{A}=\tilde{g}^{-1} d \tilde{g},
$$

with

$$
g=e^{\frac{x^{+}}{2}\left(L_{1}+L_{-1}\right)} b(\rho), \quad \quad \tilde{g}=e^{-\frac{x^{-}}{2}\left(L_{1}+L_{-1}\right)} b^{-1}(\rho) .
$$

With this rewriting we can present the isometries of $A d S_{3}$ in a very compact form and we can relate them to the $A d S_{3}$ Killing vectors and tensors. For instance, if we denote collectively the $S L(3)$ generators by $\mathcal{T}_{A}$, gauge transformations generated by the parameters

$$
\xi_{A}=g^{-1} \mathcal{T}_{A} g=\left(g^{-1} \mathcal{T}_{A} g\right)^{B} \mathcal{T}_{B}
$$

leave the $A d S_{3}$ connection $A$ invariant and similarly for $\widetilde{A}$. If we contract the gauge parameters with the inverse of the AdS dreibein, e.g.

$$
v_{a}^{\mu}=\bar{e}_{b}^{\mu}\left(g^{-1} J_{a} g\right)^{b}, \quad \quad k_{a b}^{\mu \nu}=\bar{e}_{c}^{\mu} \bar{e}_{d}^{\nu}\left(g^{-1} T_{a b} g\right)^{c d},
$$


the complete set of isometries gives rise to the 6 Killing vectors of $A d S_{3}$ and to its 10 traceless Killing tensors. The latter can be interpreted as the generators of spin-3 gauge transformations (2.36) which leave the AdS background invariant. If one acts repeatedly with the gauge transformations generated by (4.6) (or their analogues involving $\tilde{g}$ ) on a generic solution (4.3) one obtains expressions of the form

$$
\begin{array}{ll}
A_{+}=b^{-1}(\rho) a\left(x^{+}\right) b(\rho), & \widetilde{A}_{+}=0, \\
A_{-}=0, & \widetilde{A}_{-}=b(\rho) \tilde{a}\left(x^{-}\right) b^{-1}(\rho), \\
A_{\rho}=b^{-1}(\rho) \partial_{\rho} b(\rho), & \widetilde{A}_{\rho}=b(\rho) \partial_{\rho} b^{-1}(\rho),
\end{array}
$$

with $b=e^{\rho L_{0}} \cdot a\left(x^{+}\right)$and $\tilde{a}\left(x^{-}\right)$are Lie-algebra valued functions which take values in the whole $\operatorname{sl}(3)$. For instance

$$
a\left(x^{+}\right)=\sum_{i=-1}^{1} \ell^{i}\left(x^{+}\right) L_{i}+\sum_{m=-2}^{2} w^{m}\left(x^{+}\right) W_{m} .
$$

As anticipated, the flat connections (4.8) still satisfy the conditions (3.19) and (3.12).

Nevertheless, eq. (4.8) does not yet provide a satisfactory parameterisation of the space of asymptotically AdS solutions. In fact, the discussion of Section 3 makes it clear that the asymptotic symmetries of the solution space (4.8) are described by a Kac-Moody algebra. On the other hand, the most natural way to define asymptotically AdS solutions in any extension of Einstein gravity is to keep the asymptotic conformal symmetry of pure gravity and perhaps to extend it. Moreover, the procedure leading to (4.8) destroys the parameterisation (4.3) even in the pure gravity sector. This strongly suggests that eq. (4.8) should be supplemented by additional boundary conditions analogous to those introduced in [40] for pure gravity.

In the gravity sector this can be understood by noticing that diffeomorphisms actually constitute a particular class of gauge transformations: those with gauge parameters linear in the fields [14]. This enables one to recover the conformal asymptotic symmetry group of pure gravity directly in the CS formulation [32] and this still holds for higher-spin gauge transformations. For instance, by inverting eq. (2.37) (for $s=3$ ) one realises that, at the linearised level, the metric-like gauge transformations (2.36) are associated to CS gauge transformations whose parameters are quadratic in the inverse dreibein. Unfortunately, when considering non-trivial spin-3 backgrounds the identification between metric-like gauge transformations - which are the spin-3 analogue of the gravity diffeomorphism and CS gauge transformations becomes definitely more complicated.

Rather than giving a detailed characterisation of this relation in the spirit of [32], we can identify the additional boundary conditions simply by requiring that the resulting asymptotic symmetry group contains the conformal group. This can be achieved by 
looking at the Anti-de Sitter solution itself,

$$
\begin{aligned}
& A_{A d S}=b^{-1}\left(L_{1}+\frac{1}{4} L_{-1}\right) b d x^{+}+b^{-1} \partial_{\rho} b d \rho, \\
& \widetilde{A}_{A d S}=-b\left(\frac{1}{4} L_{1}+L_{-1}\right) b^{-1} d x^{-}+b \partial_{\rho} b^{-1} d \rho,
\end{aligned}
$$

and by constraining the deviation of a generic solution of the field equations from its boundary value. We thus call a solution asymptotically Anti-de Sitter if it satisfies (3.12) and (3.19) and its difference to the AdS-solution is finite at the boundary,

$$
\left.\left(A-A_{\text {AdS }}\right)\right|_{\text {boundary }}=\mathcal{O}(1)
$$

with a similar condition for $\widetilde{A}$. In the following we will mainly deal with $A$, the results for $\widetilde{A}$ follow by analogy.

In the next section we shall prove that any background which satisfies the rather natural additional boundary condition (4.11) has an asymptotic extended conformal symmetry. This result is the main motivation for choosing the boundary conditions (3.11) and (4.11) in the gauge (3.12). Notice that they are satisfied by the pure-gravity solutions (4.3) and by their supergravity extensions [41]. In Section 4.3 we shall express them as falloff conditions for the metric-like fields, thus showing their analogies with the standard Brown-Henneaux boundary conditions.

\subsection{Asymptotic symmetry algebra}

In Section 3 we have seen that, after the gauge fixing (3.12), for any CS theory the space of solutions satisfying the boundary condition $(3.11)$ is parameterised by $a(\theta)$. For $S L(3)$ we expand $a(\theta)$ in the $\left\{L_{i}, W_{m}\right\}$ basis,

$$
a(\theta)=\sum_{i=-1}^{1} \ell^{i}(\theta) L_{i}+\sum_{m=-2}^{2} w^{m}(\theta) W_{m} .
$$

The additional boundary condition (4.11) then translates into the following conditions on the components $\ell^{i}$ and $w^{m}$ :

$$
\ell^{1}=1, \quad w^{1}=w^{2}=0 .
$$

These are first-class constraints with respect to the Poisson structure (3.25) (given explicitly in Appendix B), and therefore they generate gauge transformations. We can use them to set

$$
\ell^{0}=0, \quad w^{0}=w^{-1}=0 .
$$

This completely fixes the gauge freedom [21], and the set of constraints (4.13) and (4.14) is now second class. The degrees of freedom that remain are the components $\ell^{-1}$ and $w^{-2}$. Different choices for them distinguish physically inequivalent solutions. 
We can now choose a convenient normalisation for these components and cast a generic asymptotically Anti-de Sitter solution of the field equations in the form

$$
a(\theta)=L_{1}+\frac{2 \pi}{k} \mathcal{L}(\theta) L_{-1}+\frac{\pi}{2 k \sigma} \mathcal{W}(\theta) W_{-2}
$$

This extends eq. (4.3) by the addition of the $\mathcal{W}(\theta)$ term. Below eq. (3.20) we have seen that the global symmetries of the space of solutions parameterised by $a(\theta)$ are described by global gauge transformations. We expand their parameters as

$$
\lambda(\theta)=\sum_{i=-1}^{1} \varepsilon^{i}(\theta) L_{i}+\sum_{m=-2}^{2} \chi^{m}(\theta) W_{m}
$$

and identify those that leave the structure of (4.15) invariant. They are characterised by relations between the $\varepsilon^{i}$ and $\chi^{m}$ which are conveniently expressed in terms of $\varepsilon \equiv \varepsilon^{1}$ and $\chi \equiv \chi^{2}$. Denoting $\theta$-derivatives by primes, one obtains

$$
\begin{aligned}
& \varepsilon^{0}=-\varepsilon^{\prime}, \\
& \varepsilon^{-1}=\frac{1}{2} \varepsilon^{\prime \prime}+\frac{2 \pi}{k} \varepsilon \mathcal{L}+\frac{4 \pi}{k} \chi \mathcal{W},
\end{aligned}
$$

and

$$
\begin{aligned}
& \chi^{1}=-\chi^{\prime}, \\
& \chi^{0}=\frac{1}{2} \chi^{\prime \prime}+\frac{4 \pi}{k} \chi \mathcal{L}, \\
& \chi^{-1}=-\frac{1}{6} \chi^{\prime \prime \prime}-\frac{10 \pi}{3 k} \chi^{\prime} \mathcal{L}-\frac{4 \pi}{3 k} \chi \mathcal{L}^{\prime}, \\
& \chi^{-2}=\frac{1}{24} \chi^{\prime \prime \prime \prime}+\frac{4 \pi}{3 k} \chi^{\prime \prime} \mathcal{L}+\frac{7 \pi}{6 k} \chi^{\prime} \mathcal{L}^{\prime}+\frac{\pi}{3 k} \chi \mathcal{L}^{\prime \prime}+\frac{4 \pi^{2}}{k^{2}} \chi \mathcal{L}^{2}+\frac{\pi}{2 k \sigma} \varepsilon \mathcal{W} .
\end{aligned}
$$

Under these transformations the functions $\mathcal{L}(\theta)$ and $\mathcal{W}(\theta)$ vary as

$$
\begin{aligned}
& \delta_{\varepsilon} \mathcal{L}=\varepsilon \mathcal{L}^{\prime}+2 \varepsilon^{\prime} \mathcal{L}+\frac{k}{4 \pi} \varepsilon^{\prime \prime \prime} \\
& \delta_{\varepsilon} \mathcal{W}=\varepsilon \mathcal{W}^{\prime}+3 \varepsilon^{\prime} \mathcal{W}
\end{aligned}
$$

and

$$
\begin{aligned}
\delta_{\chi} \mathcal{L}= & 2 \chi \mathcal{W}^{\prime}+3 \chi^{\prime} \mathcal{W} \\
\delta_{\chi} \mathcal{W}= & \frac{\sigma}{3}\left(2 \chi \mathcal{L}^{\prime \prime \prime}+9 \chi^{\prime} \mathcal{L}^{\prime \prime}+15 \chi^{\prime \prime} \mathcal{L}^{\prime}+10 \chi^{\prime \prime \prime} \mathcal{L}+\frac{k}{4 \pi} \chi^{(5)}\right. \\
& \left.+\frac{64 \pi}{k}\left(\chi \mathcal{L} \mathcal{L}^{\prime}+\chi^{\prime} \mathcal{L}^{2}\right)\right) .
\end{aligned}
$$

Notice that eq. (4.19b) manifests that $\mathcal{W}$ is a primary field of conformal weight 3 with respect to $\mathcal{L}$, that plays the role of energy momentum tensor for the boundary theory. 
This is actually one of the main advantages of the gauge fixing that leads to (4.14). With the convention (A.5) for the Killing metric (which is consistent with (2.24)) the charges (3.22) which generate the transformations (4.19) and (4.20) read

$$
Q(\lambda)=\int d \theta \varepsilon(\theta) \mathcal{L}(\theta)+\int d \theta \chi(\theta) \mathcal{W}(\theta) .
$$

They generate global symmetries via eq. (3.8) which allows us to identify the Poisson structure on the phase space of asymptotically Anti-de Sitter solutions. Eqs. (4.19) and (4.20) lead to

$$
\begin{aligned}
& \left\{\mathcal{L}(\theta), \mathcal{L}\left(\theta^{\prime}\right)\right\}=-\left(\delta\left(\theta-\theta^{\prime}\right) \mathcal{L}^{\prime}(\theta)+2 \delta^{\prime}\left(\theta-\theta^{\prime}\right) \mathcal{L}(\theta)\right)-\frac{k}{4 \pi} \delta^{\prime \prime \prime}\left(\theta-\theta^{\prime}\right) \\
& \left\{\mathcal{L}(\theta), \mathcal{W}\left(\theta^{\prime}\right)\right\}=-\left(2 \delta\left(\theta-\theta^{\prime}\right) \mathcal{W}^{\prime}(\theta)+3 \delta^{\prime}\left(\theta-\theta^{\prime}\right) \mathcal{W}(\theta)\right) \\
& \left\{\mathcal{W}(\theta), \mathcal{W}\left(\theta^{\prime}\right)\right\}= \\
& -\frac{\sigma}{3}\left(2 \delta\left(\theta-\theta^{\prime}\right) \mathcal{L}^{\prime \prime \prime}(\theta)+9 \delta^{\prime}\left(\theta-\theta^{\prime}\right) \mathcal{L}^{\prime \prime}(\theta)+15 \delta^{\prime \prime}\left(\theta-\theta^{\prime}\right) \mathcal{L}^{\prime}(\theta)+10 \delta^{\prime \prime \prime}\left(\theta-\theta^{\prime}\right) \mathcal{L}(\theta)\right. \\
& \left.+\frac{k}{4 \pi} \delta^{(5)}\left(\theta-\theta^{\prime}\right)+\frac{64 \pi}{k}\left(\delta\left(\theta-\theta^{\prime}\right) \mathcal{L}(\theta) \mathcal{L}^{\prime}(\theta)+\delta^{\prime}\left(\theta-\theta^{\prime}\right) \mathcal{L}^{2}(\theta)\right)\right)
\end{aligned}
$$

This is the classical $\mathcal{W}_{3}$-algebra (see e.g. [43]) with central charge

$$
c=6 k=\frac{3 l}{2 G}
$$

which is the same as for pure gravity [9].

An alternative way to present the $\mathcal{W}_{3}$-algebra is in terms of the Fourier modes of $\mathcal{L}$ and $\mathcal{W}$ which are defined as

$$
\mathcal{L}(\theta)=-\frac{1}{2 \pi} \sum_{p \in \mathbb{Z}} \mathcal{L}_{p} e^{-i p \theta}, \quad \mathcal{W}(\theta)=\frac{1}{2 \pi} \sum_{p \in \mathbb{Z}} \mathcal{W}_{p} e^{-i p \theta}
$$

If we shift the $\mathcal{L}$ zero mode according to

$$
\mathcal{L}_{p} \rightarrow \mathcal{L}_{p}-\frac{k}{4} \delta_{p, 0}
$$

and use $c=6 k$, we obtain ${ }^{8}$

$$
\begin{aligned}
i\left\{\mathcal{L}_{p}, \mathcal{L}_{q}\right\} & =(p-q) \mathcal{L}_{p+q}+\frac{c}{12}\left(p^{3}-p\right) \delta_{p+q, 0}, \\
i\left\{\mathcal{L}_{p}, \mathcal{W}_{q}\right\} & =(2 p-q) \mathcal{W}_{p+q}, \\
i\left\{\mathcal{W}_{p}, \mathcal{W}_{q}\right\}=-\frac{\sigma}{3}\left[(p-q)\left(2 p^{2}+2 q^{2}-p q-8\right) \mathcal{L}_{p+q}+\frac{96}{c}(p-q) \Lambda_{p+q}\right. & \\
& \left.\quad+\frac{c}{12} p\left(p^{2}-1\right)\left(p^{2}-4\right) \delta_{p+q, 0}\right],
\end{aligned}
$$

\footnotetext{
${ }^{8}$ This differs from eq. (19) of [43] by a rescaling of the $\mathcal{W}_{n}$ by a factor of $\sqrt{10}$.
} 
where we have defined

$$
\Lambda_{p} \equiv \sum_{q \in \mathbb{Z}} \mathcal{L}_{p+q} \mathcal{L}_{-q}
$$

The same algebra is obtained for each of the two $S L(3)$ CS theories which comprise the action (2.23). Therefore, the asymptotic symmetry of a spin-3 field coupled to gravity which is asymptotically AdS generate the $\mathcal{W}_{3} \otimes \mathcal{W}_{3}$ algebra.

The approach we followed in deriving (4.26) is the one used e.g. in [41]. We now present, following [33], an alternative derivation by explicitly computing the Dirac brackets of the generators of the algebra. If we collectively denote the second-class constrains (4.13) and (4.14) by $\left\{\chi_{\alpha} \approx 0\right\}$, we need to compute, using (3.25), the (non-degenerate) matrix $C_{\alpha \beta}=\left\{\chi_{\alpha}, \chi_{\beta}\right\}$. The Dirac bracket between two phase-space functions $f, g$ on the constraint surface is

$$
\{f, g\}_{*}=\{f, g\}-\left\{f, \chi_{\alpha}\right\}\left(C^{-1}\right)^{\alpha \beta}\left\{\chi_{\beta}, g\right\} .
$$

We work directly with the Fourier modes of $\ell^{m}(\theta)$ and $w^{n}(\theta)$, defined as

$$
\ell^{m}(\theta)=\frac{1}{k} \sum_{p \in \mathbb{Z}} \ell_{p}^{m} e^{-i p \theta} \quad, \quad w^{n}(\theta)=\frac{1}{k} \sum_{p \in \mathbb{Z}} w_{p}^{n} e^{-i p \theta} .
$$

In terms of Fourier modes, the constraints (4.13) and (4.14) read

$$
\begin{aligned}
\ell_{p}^{1} & \approx k \delta_{p, 0}, & \ell_{p}^{0} & \approx 0, \\
w_{p}^{2} & \approx 0, & w_{p}^{1} & \approx 0, \\
w_{p}^{0} & \approx 0, & w_{p}^{-1} & \approx 0 .
\end{aligned}
$$

There are infinitely many of them and the matrix $C$ decomposes into matrix blocks of infinite size,

$$
C=\frac{1}{\sigma}\left(\begin{array}{cccccc}
0 & 2 \sigma k \delta_{p+q, 0} & 0 & 0 & 0 & 0 \\
-2 \sigma k \delta_{p+q, 0} & 2 i p \sigma k \delta_{p+q, 0} & 0 & 0 & 0 & 0 \\
0 & 0 & 0 & 0 & 0 & k \delta_{p+q, 0} \\
0 & 0 & 0 & 0 & -3 k \delta_{p+q, 0} & i p k \delta_{p+q, 0} \\
0 & 0 & 0 & 3 k \delta_{p+q, 0} & -\frac{3 i p k}{2} \delta_{p+q, 0} & -3 \ell_{p+q}^{-1} \\
0 & 0 & -k \delta_{p+q, 0} & -i p k \delta_{p+q, 0} & 3 \ell_{p+q}^{-1} & 0
\end{array}\right) .
$$

To obtain this we need the brackets between the modes which we collected in Appendix B. For instance, the second entry in the first row is determined from the block

$$
\left\{\ell_{p}^{1}, \ell_{q}^{0}\right\}=2 \ell_{p+q}^{1} \approx 2 k \delta_{p+q, 0}
$$

of eq. (B.1). The inverse of $C$ can be determined to be

$$
C^{-1}=\left(\begin{array}{cccccc}
-\frac{i p}{2 k} \delta & -\frac{1}{2 k} \delta & 0 & 0 & 0 & 0 \\
\frac{1}{2 k} \delta & 0 & 0 & 0 & 0 & 0 \\
0 & 0 & \frac{-\sigma i(p-q)}{k^{2}} \ell_{-p-q}^{-1}+\frac{\sigma i p^{3}}{6 k} \delta & -\frac{\sigma}{k^{2}} \ell_{-p-q}^{-1}+\frac{\sigma p^{2}}{6 k} \delta & -\frac{\sigma i p}{3 k} \delta & -\frac{\sigma}{k} \delta \\
0 & 0 & \frac{\sigma}{k^{2}} \ell_{-p-q}^{-1}-\frac{\sigma p^{2}}{6 k} \delta & \frac{\sigma i p}{6 k} \delta & \frac{\sigma}{3 k} \delta & 0 \\
0 & 0 & -\frac{\sigma i p}{3 k} \delta & -\frac{\sigma}{3 k} \delta & 0 & 0 \\
0 & 0 & \frac{\sigma}{k} \delta & 0 & 0 & 0
\end{array}\right),
$$


where $\delta$ stands for $\delta_{p+q, 0}$. From that we find the following induced Poisson structure,

$$
\begin{aligned}
i\left\{\ell_{p}^{-1}, \ell_{q}^{-1}\right\}_{*}= & (p-q) \ell_{p+q}^{-1}-\frac{k}{2} p^{3} \delta_{p+q, 0}, \\
i\left\{\ell_{p}^{-1}, w_{q}^{-2}\right\}_{*}= & (2 p-q) w_{p+q}^{-2}, \\
i\left\{w_{p}^{-2}, w_{q}^{-2}\right\}_{*}= & \frac{k p^{5}}{96 \sigma} \delta_{p+q, 0}-\frac{1}{48 \sigma}(p-q)\left(2 p^{2}+2 q^{2}-p q\right) \ell_{p+q}^{-1} \\
& +\frac{1}{3 \sigma k}(p-q) \sum_{p^{\prime}} \ell_{p+q+p^{\prime}}^{-1} \ell_{-p^{\prime}}^{-1} .
\end{aligned}
$$

This is again the classical $\mathcal{W}_{3}$-algebra with central charge $c=6 k$. In fact, it can be related to $(4.26)$ by identifying

$$
\ell_{p}^{-1} \rightarrow-\mathcal{L}_{-p}+\frac{k}{4} \delta_{p, 0}, \quad w_{p}^{-2} \rightarrow \frac{1}{4 \sigma} \mathcal{W}_{-p}
$$

\subsection{Fall-off conditions for the metric-like fields}

In Section 4.1 we identified asymptotically Anti-de Sitter solutions combining the condition (4.11) with (3.11) and (3.12). We can now translate this into fall-off conditions for the metric-like fields $g_{\mu \nu}$ and $\varphi_{\mu \nu \rho}$. This allows for a direct comparison with the standard pure-gravity result of Brown and Henneaux [9] that further supports our choice. To this end we first have to express the metric-like fields in terms of the vielbein-like ones. The goal is the generalisation of the pure-gravity identity $g_{\mu \nu}=\eta_{a b} e_{\mu}{ }^{a} e_{\nu}{ }^{b}$ and of the relation (2.12) which is valid at the linearised level. The rationale behind both expressions is their invariance under local Lorentz-like gauge transformations. Therefore, we can look for their full non-linear analogues by imposing the invariance under the gauge transformations (2.55) and (2.56) generated by $\Lambda^{a}$ and $\Lambda^{a b}$. This fixes the structure of the metric-like fields up to a normalisation factor. For $\sigma=\tilde{\sigma}$ the result is

$$
\begin{aligned}
& g=e_{a} e^{a}-2 \sigma e_{a b} e^{a b} \\
& \varphi=e_{a} e_{b} e^{a b}+\frac{4}{3} \sigma e_{a c} e_{b}^{c} e^{a b}
\end{aligned}
$$

where we omitted for brevity the form indices so that, for instance,

$$
g=\eta_{a b}\left(e_{\mu}^{a} e_{\nu}^{b}-2 \sigma \eta_{c d} e_{\mu}^{a c} e_{\nu}^{b d}\right) d x^{\mu} \otimes d x^{\nu}
$$

Notice that the definition of the metric receives a correction quadratic in the spin-3 vielbeins with respect to the pure-gravity expression. This is required by invariance under spin-3 Lorentz-like gauge transformations, while the two terms are independently invariant under the usual Lorentz transformations. The result crucially rests on the trace constraints. 
Eqs. (4.36) admit a very convenient algebraic characterisation since they can be related to the quadratic and to the cubic Casimir of $s l(3)$, respectively. In fact, these expressions can be recovered from

$$
g_{\mu \nu}=\operatorname{tr}\left[e_{(\mu} \cdot e_{\nu)}\right], \quad \quad \varphi_{\mu \nu \rho}=\frac{1}{9 \sqrt{-\sigma}} \operatorname{tr}\left[e_{(\mu} \cdot e_{\nu} \cdot e_{\rho)}\right],
$$

where

$$
e_{\mu}=e_{\mu}^{a} J_{a}+e_{\mu}^{a b} T_{a b}
$$

The relation with the $\operatorname{sl}(3)$ Casimir operators can be realised by noticing that, for any Lie algebra $\mathfrak{g}$ generated by $\left\{\mathcal{T}_{A}\right\}$, a set of independent Casimir operators can be built as

$$
C_{p}=a^{A_{1} \ldots A_{p}} \mathcal{T}_{A_{1}} \ldots \mathcal{T}_{A_{p}}
$$

where the fully symmetric $\mathfrak{g}$-invariant tensor $a^{A_{1} \ldots A_{p}}$ is defined by

$$
a_{A_{1} \ldots A_{p}} \sim \operatorname{tr}\left[\mathcal{T}_{\left(A_{1}\right.} \ldots \mathcal{T}_{\left.A_{p}\right)}\right]
$$

and indices are lowered and raised with the Killing metric $\gamma_{A B}$ [44]. The metric-like spin-3 field $\varphi_{\mu \nu \rho}$ is thus obtained from the contraction of the vielbeins with the symmetric rank-3 invariant tensor of $s l(3)$, in full analogy with the relation between the Riemannian metric $g_{\mu \nu}$ and the Killing metric.

Eqs. (4.38) provide an intrinsic representation of the metric-like fields. We can use them to express the metric-like fields in terms of the unconstrained potentials $\hat{e}, E$ associated to the $\left\{L_{i}, W_{m}\right\}$ basis. Substituting

$$
e_{\mu}=\hat{e}_{\mu}^{i} L_{i}+E_{\mu}^{m} W_{m}
$$

into eqs. (4.38) we find for the metric

$$
g=-4 \hat{e}^{1} \hat{e}^{-1}+\hat{e}^{0} \hat{e}^{0}-\frac{4}{3} \sigma\left(12 E^{2} E^{-2}-3 E^{1} E^{-1}+E^{0} E^{0}\right)
$$

and for the spin-3 field

$$
\begin{aligned}
& \varphi=\{ 4\left(\hat{e}^{-1} \hat{e}^{-1} E^{2}+\hat{e}^{1} \hat{e}^{1} E^{-2}\right)+\frac{4}{3} \hat{e}^{-1} \hat{e}^{1} E^{0}+\frac{2}{3} \hat{e}^{0} \hat{e}^{0} E^{0} \\
&\left.-2 \hat{e}^{0}\left(\hat{e}^{-1} E^{1}+\hat{e}^{1} E^{-1}\right)\right\} \\
&+\frac{4}{3} \sigma\left\{3\left(E^{-1} E^{-1} E^{2}+E^{1} E^{1} E^{-2}\right)-8 E^{-2} E^{0} E^{2}-E^{-1} E^{0} E^{1}+\frac{2}{9} E^{0} E^{0} E^{0}\right\} .
\end{aligned}
$$

In order to identify the fall-off conditions for the metric-like fields one now has to substitute in eqs. (4.43) and (4.44) the form of the general asymptotically AdS solution of the field equations that was presented in (4.15) in terms of the frame-like fields. Choosing 
for $\widetilde{A}$ the same normalisation of eq. (4.15) but with a different overall sign, the result for the metric reads

$$
\begin{aligned}
g & =l^{2} \frac{d r^{2}}{r^{2}}-\left\{r^{2}+(8 \pi G l)^{2}\left(\frac{\mathcal{L}\left(x^{+}\right) \widetilde{\mathcal{L}}\left(x^{-}\right)}{r^{2}}+\frac{l^{2}}{4 \sigma} \frac{\mathcal{W}\left(x^{+}\right) \widetilde{\mathcal{W}}\left(x^{-}\right)}{r^{4}}\right)\right\} d x^{+} d x^{-} \\
& -8 \pi G l\left(\mathcal{L}\left(x^{+}\right)\left(d x^{+}\right)^{2}+\widetilde{\mathcal{L}}\left(x^{-}\right)\left(d x^{-}\right)^{2}\right)
\end{aligned}
$$

where with respect to eq. (4.1) we performed the change of coordinates $\rho=\log (r / l)$. For the spin-3 field one obtains

$$
\begin{aligned}
\varphi & =\frac{l}{8 \sigma}(8 \pi G l)\left(\mathcal{W}\left(x^{+}\right)\left(d x^{+}\right)^{3}+\widetilde{\mathcal{W}}\left(x^{-}\right)\left(d x^{-}\right)^{3}\right) \\
& +\frac{l}{8 \sigma}(8 \pi G l)^{2}\left(2 \frac{\widetilde{\mathcal{L}}\left(x^{-}\right) \mathcal{W}\left(x^{+}\right)}{r^{2}}+(8 \pi G l) \frac{\mathcal{L}\left(x^{+}\right)^{2} \widetilde{\mathcal{W}}\left(x^{-}\right)}{r^{4}}\right)\left(d x^{+}\right)^{2} d x^{-} \\
& +\frac{l}{8 \sigma}(8 \pi G l)^{2}\left(2 \frac{\mathcal{L}\left(x^{+}\right) \widetilde{\mathcal{W}}\left(x^{-}\right)}{r^{2}}+(8 \pi G l) \frac{\widetilde{\mathcal{L}}\left(x^{-}\right)^{2} \mathcal{W}\left(x^{+}\right)}{r^{4}}\right)\left(d x^{-}\right)^{2} d x^{+}
\end{aligned}
$$

Since eq. (4.15) solves eqs. (2.53) and (2.54), these expressions provide exact solutions of their corresponding second-order field equations, whose precise form is still to be determined. However, it is also interesting to look at the leading behaviour of eqs. (4.45) and (4.46). For instance, recognising that the metric appears in the Fefferman-Graham gauge [39]

$$
g=l^{2} \frac{d r^{2}}{r^{2}}+r^{2} g_{i j} d x^{i} d x^{j},
$$

one observes that at leading order the spatial metric satisfies the usual condition

$$
g_{i j}=\eta_{i j}+\mathcal{O}\left(1 / r^{2}\right)
$$

identifying an asymptotically $A d S_{3}$ solution with a flat boundary metric. In this sense the boundary conditions of eq. (4.11) can be understood as those that do not spoil the usual fall-off conditions for the metric identified long ago by Brown and Henneaux [9]. In fact, the Fefferman-Graham asymptotic conditions (4.48) coincide with the BrownHenneaux ones up to residual boundary diffeomorphism. The full gauge fixing leading to (4.14) indeed implies that eqs. (4.45) and (4.46) provide a fully gauge-fixed version of the admissible fall-off conditions (where the gauge fixing is meant to be performed with respect both to residual boundary diffeomorphisms and to residual boundary spin-3 gauge transformations).

Notice also that the expressions for the metric-like fields can be cast in the form

$$
\begin{aligned}
g & =l^{2} \frac{d r^{2}}{r^{2}}+\left(r^{2} \eta_{i j} d x^{i} d x^{j}-\mathcal{L}_{i j}\left(x^{m}\right) d x^{i} d x^{j}\right)+\mathcal{O}\left(r^{-2}\right) \\
\varphi & =\mathcal{W}_{i j k}\left(x^{m}\right) d x^{i} d x^{j} d x^{k}+\mathcal{O}\left(r^{-2}\right)
\end{aligned}
$$


where the tensors $\mathcal{L}_{i j}$ and $\mathcal{W}_{i j k}$ are traceless and conserved. In fact, in two-dimensions these conditions imply that they only have two independent components, one left-moving and one right-moving. In the boundary theory, which is defined on a flat background, these two objects are thus the Noether currents associated to the extended conformal symmetry we discovered in (4.22).

\section{Comments on higher rank groups}

In Section 2.2 we have shown how one can use $S L(n) \times S L(n)$ CS theories to describe a particular class of higher-spin interactions. The corresponding spectrum was identified simply by looking at what generators $T_{a_{1} \ldots a_{s-1}}$ one needs in order to describe a $\operatorname{sl}(n)$ algebra. To each $T_{a_{1} \ldots a_{s-1}}$ one can then associate a spin-s field. To this end, it is crucial to realise that the commutator (2.21) implies that the independent components of $T_{a_{1} \ldots a_{s-1}}$ transform in the $(2 s-1)$-dimensional irreducible representation under the adjoint action of $\operatorname{sl}(2, \mathbb{R})$. It is thus natural to associate a three-dimensional higher-spin bosonic gauge theory to any CS theory based on a $G \times G$ gauge group. The inclusion of fermions - which we do not discuss in the present paper - will be obtained by considering also supergroups. The selection of an embedding of $\operatorname{sl}(2, \mathbb{R})$ in $\mathfrak{g}$ then induces a branching of the generators in sets that transform irreducibly under the adjoint action of $\operatorname{sl}(2, \mathbb{R})$. This should determine the spectrum. As in eq. (2.47), one could then associate the combinations

$$
\mathcal{P}_{A}=\frac{1}{l}\left(\mathcal{T}_{A}-\widetilde{\mathcal{T}}_{A}\right), \quad \mathcal{M}_{A}=\mathcal{T}_{A}+\widetilde{\mathcal{T}}_{A}
$$

of the generators $\left\{\mathcal{T}_{A}\right\}$ and $\left\{\widetilde{\mathcal{T}}_{A}\right\}$ of the two copies of $\mathfrak{g}$ to vielbein-like and auxiliary fields, respectively. This procedure was indeed proposed in a number of papers dealing with higher-spin gauge fields in three space-time dimensions [16, 45, 18].

However, in the $S L(n) \times S L(n)$ case we can go beyond this identification and also control the elimination of auxiliary fields and the recovering of the Fronsdal metric-like formulation. For this reason, we shall mainly discuss the asymptotic symmetries emerging in this class of examples, and in a generalisation that we shall describe in a moment. First of all, notice that the procedure of Section 2.2 selected not only the $s l(n)$ gauge algebra, but also a particular embedding of $s l(2, \mathbb{R})$ in it: the principal embedding. For a generic simple Lie algebra $\mathfrak{g}$, the principal embedding has the property that the spins occurring in the decomposition of $\mathfrak{g}$ into $\operatorname{sl}(2, \mathbb{R})$ representations are $\left(l_{i}\right)$, where $l_{i}, i=1, \ldots, r=$ $\operatorname{rank}(\mathfrak{g})$, are the exponents of the algebra $[46]$ and $\left(l_{i}+1\right)$ are the ranks of the independent Casimir operators of $\mathfrak{g}$. This suggests to extend the identification of Section 4.3 between metric-like fields and $\mathfrak{g}$-invariant tensors also to the $S L(n) \times S L(n)$ models of Section 2.2 and, in more generality, to all higher-spin gauge theories obtained via the principal embedding of $\operatorname{sl}(2, \mathbb{R})$ into a simple $\mathfrak{g}$. Therefore, in the following we shall focus on this class of higher-spin gauge theories, which provide the most natural generalisation of the spin-3 example that we discussed in detail in Section 4. 
In order to discuss asymptotic symmetries in this context, let us recall that if one imposes the boundary condition (3.11), then the boundary dynamics of the CS theory is described by a Wess-Zumino-Witten model (see, for instance, the reviews [33, 34] and references therein). Furthermore, the additional conditions (4.13) are those inducing the Hamiltonian reduction of the WZW model to a $S L(3)$ Toda theory [21]. At this purely algebraic level, the reduction of the affine algebra (3.25) to the $\mathcal{W}_{3}$-algebra (4.26) is often called Drinfeld-Sokolov (DS) reduction. This is a general procedure that we can apply even beyond the spin-3 case. Consider a generic $G \times G$ CS theory of the type just discussed (i.e. characterised by the principal embedding of $\operatorname{sl}(2, \mathbb{R})$ into $\mathfrak{g})$. We can still fix the gauge as in eq. (3.12) and impose the natural condition

$$
\left.\left(A-A_{\mathrm{AdS}}\right)\right|_{\text {boundary }}=\mathcal{O}(1) .
$$

This extends the characterisation of asymptotically Anti-de Sitter solutions in eq. (4.11) to the general case. We can also impose the boundary condition (3.11) and expand the function $a(\theta)$ of eq. (3.17) as

$$
a(\theta)=\sum_{i=-1}^{1} \ell^{i}(\theta) L_{i}+\sum_{i=2}^{r} \sum_{m=-l_{i}}^{l_{i}} w^{l_{i}, m}(\theta) W_{l_{i}, m} .
$$

The first term is related to the lowest exponent $l_{1}$ which is always one. The $L_{i}$ are the $s l(2, \mathbb{R})$ generators while the $W_{l, m}$ generators are those transforming in the spin- $l$ representation under the adjoint action of $s l(2, \mathbb{R})$ (which one associates with spin- $(l+1)$ gauge fields). Eq. (5.2) then leads to the following constraints on the components of $a(\theta)$ :

$$
\ell^{1}=1, \quad w^{l, m}=0 \quad \forall l \text { and } \forall m>0 .
$$

We can use these first class constraints to reach the so called highest-weight gauge [21] which is characterised by the additional conditions

$$
\ell^{0}=0, \quad w^{l, m}=0 \quad \forall l \text { and } \forall m>-l+1 .
$$

The remaining components $w^{l,-l}(\theta)$, which are conformal primary fields of weight $l+1$ with respect to $\ell^{-1} \sim \mathcal{L}$, enter $a(\theta)$ contracted with $W_{l,-l}$ generators. The asymptotic symmetries of these $G \times G$ CS theories are two copies of a $\mathcal{W}$-algebra determined by $G$. In particular, for the $S L(n) \times S L(n)$ class we get two copies of the $\mathcal{W}_{n}$-algebra. In fact, with (5.4) we have recovered the constraints inducing the DS reduction of the affine Lie algebra identified in Section 3 [21] and inspection of the results of [21] suffices to arrive at this conclusion about the symmetry algebra. The metric-like fields are presumably constructed as in Section 4.3 and they are in 1-1 correspondence with the $\operatorname{rank}(\mathfrak{g})$ Casimir invariants on $\mathfrak{g}$. This is reminiscent of the generalised Sugawara construction in [47].

The $\mathcal{W}$-algebras which arise as asymptotic symmetry algebras have a central charge which, as we now demonstrate, has the same value as in the case of pure gravity. Recall that in eq. (4.15) we parameterised the space of asymptotically AdS solutions by simply 
adding the function $\mathcal{W}(\theta)$ to the pure-gravity expression (4.3). It is therefore not surprising that we recovered the Brown-Henneaux value for the central charge in (4.22). A similar parameterisation of the space of asymptotically AdS solutions is obtained in the general case. The higher spin fields do not modify the structure of the pure gravity part of $a(\theta)$ and therefore the DS reduction leads to a $\mathcal{W}$-algebra whose central charge has the Brown-Henneaux value.

In terms of CS data, the value of the central charge which arises in the DS reduction is (see eqs. (2.27) and (3.1) in [21])

$$
c=12 k \operatorname{tr}\left(L_{0}^{2}\right) .
$$

Comparing the Einstein-Hilbert action and the gravity sector of (2.23) leads to the identification

$$
c=12 k \operatorname{tr}\left(L_{0}^{2}\right)=\frac{3 l}{2 G}
$$

Eq. (5.6) holds also for DS reductions performed with respect to different embeddings (see for instance the classical, $k \rightarrow \infty$, limit of eq. (78) of [48]). But, as we already stressed, if we choose a different embedding we loose the suggestive correspondence between the spectrum of the theory and the Casimir operators of the underlying algebra which played a crucial role in Section 4.3 .

\section{Conclusions}

In this paper we studied the asymptotic symmetries of asymptotically Anti-de Sitter solutions of higher-spin gauge theories coupled to three-dimensional gravity with a negative cosmological constant. We focussed on the case where only a finite number of bosonic higher-spin fields is involved, for which we showed that the asymptotic symmetries are described by two copies of a $\mathcal{W}$-algebra selected by the spectrum of the theory. These higher-spin models correspond to CS theories based on a generic finite-dimensional $G \times G$ gauge group. In particular, we discussed in detail the $S L(3) \times S L(3)$ example, describing the coupling of a spin-3 gauge field to gravity. In this case we identified a $\mathcal{W}_{3} \otimes \mathcal{W}_{3}$ algebra of asymptotic symmetries. We also showed explicitly how to relate the $S L(n) \times S L(n)$ CS theories to the standard frame-like formulation of the higher-spin dynamics. Finally, we noticed that the boundary conditions which select asymptotically AdS solutions in the $S L(3) \times S L(3)$ example coincide with the constraints inducing the Drinfeld-Sokolov reduction of a suitable $s l(3)$ affine Lie algebra to $\mathcal{W}_{3}$. Working in this framework we then discussed $G \times G$ higher-spin gauge theories based on simple Lie algebras $\mathfrak{g}$ where the gravitational sector is singled out by the principal embedding of $\operatorname{sl}(2, \mathbb{R})$ in $\mathfrak{g}$. In all cases the value of the central charge of the resulting $\mathcal{W}$-algebra is the same as that found by Brown-Henneaux for pure gravity.

The choice of working with a finite number of higher-spin fields was motivated by the simplicity of these models, that enabled us to discuss in detail various aspects of 
the relation between CS theories and higher-spin gauge theories (see for instance Section 4.3). However, it will be interesting to also extend our analysis to the three-dimensional higher-spin gauge theories of $[15,16]$, that contain in their spectra an infinite number of higher-spin gauge fields. ${ }^{9}$ In this respect they could provide more realistic toy models for comparisons with the Vasiliev theory [8], that describes an infinite tower of interacting higher-spin gauge fields in AdS backgrounds with $D \geq 4$. Other directions that deserve further investigations are the inclusion of fermions in the present framework and the study of CS theories built upon other than the principal embedding of $\operatorname{sl}(2, \mathbb{R})$. While different embeddings were already discussed in [48] in the framework of the DrinfeldSokolov reduction, their interpretation as higher-spin gauge theories could require some modifications with respect to the picture we have presented. The inclusion of ChanPaton factors [17] is also of interest, in particular in view of a possible relation between higher-spin theories and open strings. Another important aspect that requires further work is the characterisation of the boundary theory. At the classical level the DrinfeldSokolov reduction methods suggest that the relation between three-dimensional gravity and Liouville theory [40] can be extended to a more general relation between threedimensional higher-spin gauge theories and Toda theories.

\section{Acknowledgements}

We are grateful to M. Bañados, G. Barnich, M. Henneaux, O. Mišković, S.-J. Rey, A. Sagnotti and M. A. Vasiliev for interesting discussions and useful correspondence.

\section{A Conventions}

In this paper we adopt the mostly plus convention for the metric

$$
\eta_{a b}=(-,+,+)
$$

while the Levi-Civita symbol is defined such that

$$
\epsilon^{012}=-\epsilon_{012}=1
$$

A pair of parentheses denotes the symmetrisation of the indices it encloses, with the minimum number of terms and without any normalisation factor. For instance, if $T_{a b}$ is a symmetric tensor

$$
V_{(a} T_{b c)}=V_{a} T_{b c}+V_{b} T_{a c}+V_{c} T_{a b}
$$

\footnotetext{
${ }^{9}$ M. Henneaux and S.-J. Rey have considered this case and they have constructed a non-linear $W_{\infty}$ asymptotic symmetry algebra. We thank M.H. for informing us of their results prior to publication of $[22]$.
} 
A vertical bar signals that the symmetrisation also encompasses the indices lying between the next bar and the closing parenthesis. For instance

$$
V_{(a \mid} W_{d} T_{\mid b c)}=V_{a} W_{d} T_{b c}+V_{b} W_{d} T_{a c}+V_{c} W_{d} T_{a b} .
$$

In a similar fashion a pair of square brackets denotes the antisymmetrisation of the indices it encloses, again with the minimum number of terms and without any normalisation factor.

We normalise the Killing metric of $\operatorname{sl}(3)$ such that in the basis $\left\{\mathcal{T}_{A}\right\}=\left\{L_{i}, W_{m}\right\}$ with $i=-1, \ldots, 1, m=-2, \ldots, 2$ (see eq. (2.43)), it is given by

$$
\gamma_{A B}=\left(\begin{array}{cccccccc}
0 & 0 & -1 & 0 & 0 & 0 & 0 & 0 \\
0 & 1 / 2 & 0 & 0 & 0 & 0 & 0 & 0 \\
-1 & 0 & 0 & 0 & 0 & 0 & 0 & 0 \\
0 & 0 & 0 & 0 & 0 & 0 & 0 & -4 \sigma \\
0 & 0 & 0 & 0 & 0 & 0 & \sigma & 0 \\
0 & 0 & 0 & 0 & 0 & -\frac{2}{3} \sigma & 0 & 0 \\
0 & 0 & 0 & 0 & \sigma & 0 & 0 & 0 \\
0 & 0 & 0 & -4 \sigma & 0 & 0 & 0 & 0
\end{array}\right) .
$$

The invariant form which is used to write the CS action (2.23) is defined as

$$
\operatorname{tr}\left(\mathcal{T}_{A} \mathcal{T}_{B}\right)=\gamma_{A B}
$$

For the $\operatorname{sl}(2)$ generators in the basis $J_{a}$ this reproduces (2.24). This convention preserves the standard pure-gravity relation (2.25) between the level $k$ of the CS theory and the cosmological constant.

A possible $3 \times 3$ matrix realisation of the algebra $(2.43)$ is given by the fundamental representation of $\operatorname{sl}(3)$,

$$
\begin{array}{rlrl}
L_{1}= & \left(\begin{array}{lll}
0 & 0 & 0 \\
1 & 0 & 0 \\
0 & 1 & 0
\end{array}\right), & L_{-1}=\left(\begin{array}{ccc}
0 & -2 & 0 \\
0 & 0 & -2 \\
0 & 0 & 0
\end{array}\right) \\
L_{0}=\left(\begin{array}{ccc}
1 & 0 & 0 \\
0 & 0 & 0 \\
0 & 0 & -1
\end{array}\right), & W_{0}=\frac{2}{3} \sqrt{-\sigma}\left(\begin{array}{ccc}
1 & 0 & 0 \\
0 & -2 & 0 \\
0 & 0 & 1
\end{array}\right) \\
W_{1}=\sqrt{-\sigma}\left(\begin{array}{ccc}
0 & 0 & 0 \\
1 & 0 & 0 \\
0 & -1 & 0
\end{array}\right), & W_{-1}=\sqrt{-\sigma}\left(\begin{array}{ccc}
0 & -2 & 0 \\
0 & 0 & 2 \\
0 & 0 & 0
\end{array}\right) \\
W_{2}=2 \sqrt{-\sigma}\left(\begin{array}{ccc}
0 & 0 & 0 \\
0 & 0 & 0 \\
1 & 0 & 0
\end{array}\right), & W_{-2}=2 \sqrt{-\sigma}\left(\begin{array}{ccc}
0 & 0 & 4 \\
0 & 0 & 0 \\
0 & 0 & 0
\end{array}\right)
\end{array}
$$

Notice that the representatives of the $W_{m}$ generators are real for $\sigma<0$, in agreement with the association of the real form $\operatorname{sl}(3, \mathbb{R})$ with negative values of $\sigma$. Furthermore, comparison with (A.5) reveals that in the fundamental representation 'tr' in (A.6) denotes one quarter times the matrix trace. 


\section{B Useful formulae}

The non-vanishing Dirac brackets (3.25) for the $s l(3)$ modes (4.29) read

$$
\begin{aligned}
& \left\{\ell_{p}^{1}, \ell_{q}^{0}\right\}=2 \ell_{p+q}^{1} \quad\left\{\ell_{p}^{1}, \ell_{q}^{-1}\right\}=\ell_{p+q}^{0}-i p k \delta_{p+q, 0} \\
& \left\{\ell_{p}^{0}, \ell_{q}^{-1}\right\}=2 \ell_{p+q}^{-1} \quad\left\{\ell_{p}^{0}, \ell_{q}^{0}\right\}=2 i p k \delta_{p+q, 0} \\
& \left\{w_{p}^{1}, \ell_{q}^{1}\right\}=-4 w_{p+q}^{2} \quad\left\{w_{p}^{2}, \ell_{q}^{0}\right\}=4 w_{p+q}^{2} \quad\left\{w_{p}^{2}, \ell_{q}^{-1}\right\}=w_{p+q}^{1} \\
& \left\{w_{p}^{0}, \ell_{q}^{1}\right\}=-3 w_{p+q}^{1} \quad\left\{w_{p}^{1}, \ell_{q}^{0}\right\}=2 w_{p+q}^{1} \quad\left\{w_{p}^{1}, \ell_{q}^{-1}\right\}=2 w_{p+q}^{0} \\
& \left\{w_{p}^{-1}, \ell_{q}^{1}\right\}=-2 w_{p+q}^{0} \quad\left\{w_{p}^{-1}, \ell_{q}^{0}\right\}=-2 w_{p+q}^{-1} \quad\left\{w_{p}^{0}, \ell_{q}^{-1}\right\}=3 w_{p+q}^{-1} \\
& \left\{w_{p}^{-2}, \ell_{q}^{1}\right\}=-w_{p+q}^{-1} \quad\left\{w_{p}^{-2}, \ell_{q}^{0}\right\}=-4 w_{p+q}^{-2} \quad\left\{w_{p}^{-1}, \ell_{q}^{-1}\right\}=4 w_{p+q}^{-2} \\
& \left\{w_{p}^{2}, w_{q}^{-1}\right\}=\frac{1}{\sigma} \ell_{p+q}^{1} \quad\left\{w_{p}^{2}, w_{q}^{-2}\right\}=\frac{1}{2 \sigma} \ell_{p+q}^{0}-\frac{i p k}{4 \sigma} \delta_{p+q, 0} \\
& \left\{w_{p}^{1}, w_{q}^{0}\right\}=-\frac{3}{\sigma} \ell_{p+q}^{1} \quad\left\{w_{p}^{1}, w_{q}^{-1}\right\}=-\frac{1}{\sigma} \ell_{p+q}^{0}+\frac{i p k}{\sigma} \delta_{p+q, 0} \\
& \left\{w_{p}^{-1}, w_{q}^{0}\right\}=\frac{3}{\sigma} \ell_{p+q}^{-1} \quad\left\{w_{p}^{0}, w_{q}^{0}\right\}=-\frac{3 i p k}{2 \sigma} \delta_{p+q, 0} \\
& \left\{w_{p}^{-2}, w_{q}^{1}\right\}=-\frac{1}{\sigma} \ell_{p+q}^{-1}
\end{aligned}
$$

\section{References}

[1] C. Fronsdal, Phys. Rev. D 18 (1978) 3624.

[2] X. Bekaert, N. Boulanger and P. Sundell, arXiv:1007.0435 [hep-th].

[3] M. A. Vasiliev, Int. J. Mod. Phys. D 5 (1996) 763 [arXiv:hep-th/9611024];

X. Bekaert, S. Cnockaert, C. Iazeolla and M. A. Vasiliev, arXiv:hep-th/0503128;

C. Iazeolla, arXiv:0807.0406 [hep-th].

[4] D. Francia and A. Sagnotti, Class. Quant. Grav. 20 (2003) S473 [arXiv:hepth/0212185];

D. Sorokin, AIP Conf. Proc. 767 (2005) 172 [arXiv:hep-th/0405069];

N. Bouatta, G. Compère and A. Sagnotti, arXiv:hep-th/0409068;

D. Francia and A. Sagnotti, J. Phys. Conf. Ser. 33 (2006) 57 [arXiv:hep-th/0601199];

A. Campoleoni, Riv. Nuovo Cim. 33 (2010) 123 [arXiv:0910.3155 [hep-th]].

[5] A. Sagnotti, E. Sezgin and P. Sundell, arXiv:hep-th/0501156;

A. Fotopoulos and M. Tsulaia, Int. J. Mod. Phys. A 24 (2009) 1 [arXiv:0805.1346 [hep-th]].

[6] C. Aragone and S. Deser, Phys. Lett. B 86 (1979) 161. 
[7] C. Aragone and S. Deser, Class. Quant. Grav. 1 (1984) L9.

[8] M. A. Vasiliev, Phys. Lett. B 243 (1990) 378, Phys. Lett. B 567 (2003) 139 [arXiv:hep-th/0304049].

[9] J. D. Brown and M. Henneaux, Commun. Math. Phys. 104 (1986) 207.

[10] M. Bañados, C. Teitelboim and J. Zanelli, Phys. Rev. Lett. 69 (1992) 1849 [arXiv:hep-th/9204099];

M. Bañados, M. Henneaux, C. Teitelboim and J. Zanelli, Phys. Rev. D 48 (1993) 1506 [arXiv:gr-qc/9302012].

[11] A. Strominger, JHEP 9802 (1998) 009 [arXiv:hep-th/9712251].

[12] P. Bouwknegt and K. Schoutens, Phys. Rept. 223 (1993) 183 [arXiv:hep-th/9210010].

[13] A. Achúcarro and P. K. Townsend, Phys. Lett. B 180 (1986) 89.

[14] E. Witten, Nucl. Phys. B 311 (1988) 46.

[15] M. P. Blencowe, Class. Quant. Grav. 6 (1989) 443.

[16] E. Bergshoeff, M. P. Blencowe and K. S. Stelle, Commun. Math. Phys. 128 (1990) 213.

[17] M. A. Vasiliev, Int. J. Mod. Phys. A 6 (1991) 1115.

[18] S. F. Prokushkin and M. A. Vasiliev, Nucl. Phys. B 545 (1999) 385 [arXiv:hepth/9806236].

[19] A. Bilal, V. V. Fock and I. I. Kogan, Nucl. Phys. B 359 (1991) 635.

[20] J. de Boer and J. Goeree, Nucl. Phys. B 381 (1992) 329 [arXiv:hep-th/9112060].

[21] J. Balog, L. Fehér, L. O’Raifeartaigh, P. Forgács and A. Wipf, Annals Phys. 203 (1990) 76.

[22] M. Henneaux and S. J. Rey, arXiv:1008.4579 [hep-th].

[23] T. Curtright, Phys. Lett. B 85 (1979) 219.

[24] C. Fronsdal, Phys. Rev. D 20 (1979) 848.

[25] B. Binegar, J. Math. Phys. 23 (1982) 1511.

[26] M. A. Vasiliev, Yad. Fiz. 32 (1980) 855 [Sov. J. Nucl. Phys. 32 (1980) 439].

[27] M. A. Vasiliev, Fortsch. Phys. 35 (1987) 741;

V. E. Lopatin and M. A. Vasiliev, Mod. Phys. Lett. A 3 (1988) 257. 
[28] M. Hamermesh, Group theory and its applications to physical problems, Dover Publications, New York (1969).

[29] E. S. Fradkin and M. A. Vasiliev, Annals Phys. 177 (1987) 63.

[30] J. Hoppe, M.I.T. PhD Thesis (1982).

[31] A. Achúcarro and P. K. Townsend, Phys. Lett. B 229 (1989) 383.

[32] M. Bañados, Phys. Rev. D 52 (1996) 5816 [arXiv:hep-th/9405171];

M. Bañados, T. Brotz and M. E. Ortiz, Nucl. Phys. B 545 (1999) 340 [arXiv:hepth/9802076].

[33] M. Bañados, arXiv:hep-th/9901148.

[34] S. Carlip, Class. Quant. Grav. 22 (2005) R85 [arXiv:gr-qc/0503022].

[35] T. Regge and C. Teitelboim, Annals Phys. 88 (1974) 286.

[36] R. Benguria, P. Cordero and C. Teitelboim, Nucl. Phys. B 122 (1977) 61.

[37] V. E. Didenko, A. S. Matveev and M. A. Vasiliev, Theor. Math. Phys. 153 (2007) 1487 [Teor. Mat. Fiz. 153 (2007) 158] [arXiv:hep-th/0612161].

[38] K. Skenderis and S. N. Solodukhin, Phys. Lett. B 472 (2000) 316 [arXiv:hepth/9910023].

[39] C. Fefferman and C. R. Graham, "Conformal Invariants," in Elie Cartan et les Mathématiques d'aujourd'hui, Astèrisque, hors série (1985) 95.

[40] O. Coussaert, M. Henneaux and P. van Driel, Class. Quant. Grav. 12 (1995) 2961 [arXiv:gr-qc/9506019].

[41] M. Bañados, K. Bautier, O. Coussaert, M. Henneaux and M. Ortiz, Phys. Rev. D 58 (1998) 085020 [arXiv:hep-th/9805165];

M. Henneaux, L. Maoz and A. Schwimmer, Annals Phys. 282 (2000) 31 [arXiv:hepth/9910013].

[42] M. Henneaux and C. Teitelboim, Commun. Math. Phys. 98 (1985) 391.

[43] P. Mathieu, Phys. Lett. B 208 (1988) 101.

[44] S. Okubo and J. Patera, J. Math. Phys. 25 (1984) 219.

[45] C. N. Pope and P. K. Townsend, Phys. Lett. B 225 (1989) 245.

[46] A. V. Razumov and M. V. Saveliev, Lie Algebras, Geometry, and Toda-type Systems, Cambridge Lecture Notes in Physics, Cambridge University Press, Cambridge (1997). 
[47] F. A. Bais, P. Bouwknegt, M. Surridge and K. Schoutens, Nucl. Phys. B 304 (1988) 348.

[48] F. A. Bais, T. Tjin and P. van Driel, Nucl. Phys. B 357 (1991) 632. 